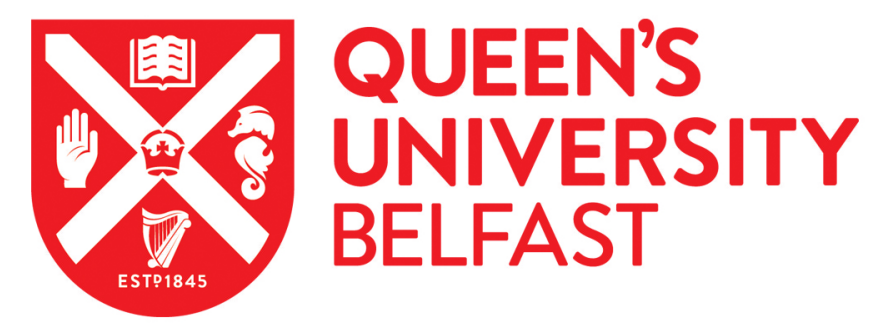

\title{
The Origin of B-type Runaway Stars: Non-LTE Abundances as a Diagnostic
}

McEvoy, C. M., Dufton, P. L., Smoker, J. V., Lambert, D. L., Keenan, F. P., Schneider, F. R. N., \& de Wit, W-J. (2017). The Origin of B-type Runaway Stars: Non-LTE Abundances as a Diagnostic. The Astrophysical Journal, 842(1), [32]. https://doi.org/10.3847/1538-4357/aa745a

\section{Published in:}

The Astrophysical Journal

Document Version:

Publisher's PDF, also known as Version of record

Queen's University Belfast - Research Portal:

Link to publication record in Queen's University Belfast Research Portal

Publisher rights

(C) 2017. The American Astronomical Society.

This work is made available online in accordance with the publisher's policies. Please refer to any applicable terms of use of the publisher.

\section{General rights}

Copyright for the publications made accessible via the Queen's University Belfast Research Portal is retained by the author(s) and / or other copyright owners and it is a condition of accessing these publications that users recognise and abide by the legal requirements associated with these rights.

Take down policy

The Research Portal is Queen's institutional repository that provides access to Queen's research output. Every effort has been made to ensure that content in the Research Portal does not infringe any person's rights, or applicable UK laws. If you discover content in the Research Portal that you believe breaches copyright or violates any law, please contact openaccess@qub.ac.uk. 


\title{
The Origin of B-type Runaway Stars: Non-LTE Abundances as a Diagnostic
}

\author{
Catherine M. McEvoy ${ }^{1,2,3}$, Philip L. Dufton ${ }^{1}$, Jonathan V. Smoker ${ }^{1,2}$, David L. Lambert ${ }^{4}$, Francis P. Keenan ${ }^{1}$, \\ Fabian R. N. Schneider ${ }^{5}$, and Willem-Jan de $\mathrm{Wit}^{2}$ \\ ${ }^{1}$ Astrophysics Research Centre, School of Mathematics and Physics, Queen's University Belfast, Belfast BT7 1NN, UK \\ 2 European Southern Observatory, Alonso de Cordova 3107, Casilla 19001, Vitacura, Santiago 19, Chile \\ ${ }^{3}$ Graduate School, King's College London, The Strand, London SE1 9NH, UK \\ ${ }^{4}$ The University of Texas at Austin, Department of Astronomy, RLM 16.316, Austin, TX 78712, USA \\ ${ }^{5}$ Department of Physics, University of Oxford, Denys Wilkinson Building, Keble Road, Oxford OX1 3RH, UK \\ Received 2017 February 17; revised 2017 May 17; accepted 2017 May 17; published 2017 June 9
}

\begin{abstract}
There are two accepted mechanisms to explain the origin of runaway OB-type stars: the binary supernova (SN) scenario and the cluster ejection scenario. In the former, an SN explosion within a close binary ejects the secondary star, while in the latter close multibody interactions in a dense cluster cause one or more of the stars to be ejected from the region at high velocity. Both mechanisms have the potential to affect the surface composition of the runaway star. TLUSTY non-LTE model atmosphere calculations have been used to determine the atmospheric parameters and the $\mathrm{C}, \mathrm{N}, \mathrm{Mg}$, and $\mathrm{Si}$ abundances for a sample of B-type runaways. These same analytical tools were used by Hunter et al. for their analysis of 50 B-type open-cluster Galactic stars (i.e., nonrunaways). Effective temperatures were deduced using the Si-ionization balance technique, surface gravities from Balmer line profiles, and microturbulent velocities derived using the $\mathrm{Si}$ spectrum. The runaways show no obvious abundance anomalies when compared with stars in the open clusters. The runaways do show a spread in composition that almost certainly reflects the Galactic abundance gradient and a range in the birthplaces of the runaways in the Galactic disk. Since the observed Galactic abundance gradients of $\mathrm{C}, \mathrm{N}, \mathrm{Mg}$, and $\mathrm{Si}$ are of a similar magnitude, the abundance ratios (e.g., $\mathrm{N} / \mathrm{Mg}$ ) are as obtained essentially uniform across the sample.
\end{abstract}

Key words: stars: atmospheres - stars: early-type - stars: rotation - stars: kinematics and dynamics

\section{Introduction}

The presence of a significant number of early-type OB mainsequence stars in the Galactic halo, as demonstrated by Greenstein \& Sargent (1974), has occasioned an extensive literature on the origins of massive young stars far from the Galactic disk, the richest site of star-forming regions. Very broadly, two explanations for halo OB main-sequence stars have survived scrutiny; both explanations consider that the stars formed in the Galactic disk and were ejected from their parental open cluster or association with sufficent velocity to reach the Galactic halo. According to these explanations, the stars deserve their common classification as "runaway" stars. A less likely explanation not considered further here is that the halo OB mainsequence stars were formed in situ (Dyson \& Hartquist 1983).

Two proposed scenarios are considered to be capable of producing runaway stars: the binary supernova $(\mathrm{SN})$ scenario (here, BSS) and the cluster ejection scenario (here, CES):

1. In the BSS proposed first by Zwicky (1957) and developed by Blaauw (1961), the runaway star was the secondary in a binary with a more massive star that experienced its terminal SN explosion. The reduced gravitational attraction of the primary's stellar remnant, either a neutron star (NS) or a black hole (BH), freed the secondary to escape with a velocity similar to its orbital velocity. The escaping secondary - the runaway starmay be accompanied by the stellar remnant.

2. In the CES proposed by Poveda et al. (1967), close encounters in a young open cluster may lead to the ejection of a star. Multibody interactions are favored to eject a single or a binary star. Simulations suggest that the most effective way to produce high-velocity runaway stars is through the interaction of two hard binary systems (Hoffer 1983). Leonard (1989) and Leonard \& Fahlman (1991) show that binary-binary interactions may result in runaway single, binary, or even merged binary stars.

As might be expected, both the BSS and the CES contribute to the runaway population. Hoogerwerf et al. (2001-see also de Zeeuw et al. 2001) use astrometric data to predict the past tracks of stars in the Galaxy to show that specific examples of runaways may be attributed to the BSS (see $\zeta$ Oph and pulsar PSR J1392+1059) or to the CES (AE Aur and $\mu$ Col ejected from Orion-see Blaauw \& Morgan (1954) and Gies \& Bolton (1986)). Across their sample of runaways, Hoogerwerf et al. (2001) estimate that two thirds arise from the BSS and one third arises from the CES. Other authors consider the CES to be the greater contributor of runaway stars.

In this paper, we provide a non-LTE analysis of $\mathrm{C}, \mathrm{N}, \mathrm{Mg}$, and $\mathrm{Si}$ abundances for a sample of runaway B stars and search for abundance differences among the sample and between the sample and B stars in young open clusters in the Galactic disk. The goal is to determine if the abundance information provides convincing evidence or even intriguing clues to the origin of a runaway. The suggestion to exploit chemical composition to judge the competing origins of runaway stars is traceable to Blaauw (1993), who proposed a study of the $\mathrm{He} / \mathrm{H}$ abundance versus the projected rotational velocity $v \sin i$ with $\mathrm{He}$ enrichment and a high $v \sin i$ resulting from the BSS. He enrichment should generally be accompanied by $\mathrm{N}$ enrichment and a parallel $\mathrm{C}$ deficiency as a result of $\mathrm{H}$-burning by the $\mathrm{CNO}$ cycles. A $\mathrm{Mg}$ and $\mathrm{Si}$ enrichment of the runaway star may result for stars provided by the BSS but not those provided by the CES. Many previous studies have reported LTE abundance analyses generally giving abundances relative to Galactic disk 
Table 1

Each Star Listed by HIP Number, Along with an Alternative Identifier

\begin{tabular}{|c|c|c|c|c|c|c|c|}
\hline HIP & Other & Vmag & $\begin{array}{l}\text { Spec. } \\
\text { Type }\end{array}$ & Obs. $^{\mathrm{a}}$ & $\begin{array}{c}v_{r} \\
\mathrm{~km} \mathrm{~s}^{-1}\end{array}$ & $\underset{\mathrm{km} \mathrm{s}^{-1}}{v \sin i}$ & Reference $^{\mathrm{b}}$ \\
\hline 2702 & HD 3175 & 9.33 & $\mathrm{~B} 4 \mathrm{~V}$ & $\mathrm{~F}$ & $-13 \pm 2$ & $26 \pm 2$ & $\mathrm{~S} 11$ \\
\hline 3812 & $\mathrm{CD}-56152$ & 10.18 & $\mathrm{~B} 2 \mathrm{~V}$ & $\mathrm{U}$ & $14 \pm 8$ & $194 \pm 7$ & S11 \\
\hline 7873 & HD 10747 & 8.15 & $\mathrm{~B} 2 \mathrm{~V}$ & $\mathrm{~F}$ & $-9 \pm 2$ & $15 \pm 1$ & $\mathrm{~T} 11$ \\
\hline 13489 & HD 18100 & 8.44 & B5II/III & $\mathrm{F}$ & $80 \pm 7$ & $241 \pm 6$ & M05 \\
\hline 16758 & HD 22586 & 9.33 & B4V & $\mathrm{F}$ & $-13 \pm 2$ & $88 \pm 3$ & $\mathrm{~S} 11$ \\
\hline 45563 & HD 78584 & 8.20 & B3 & $\mathrm{T}$ & $-120 \pm 6$ & $102 \pm 4$ & $\mathrm{~T} 11$ \\
\hline 55051 & HD 97991 & 7.41 & $\mathrm{~B} 2 / 3 \mathrm{~V}$ & $\mathrm{U}$ & $31 \pm 3$ & $135 \pm 3$ & S11 \\
\hline 56322 & HD 100340 & 10.12 & B0 & $\mathrm{T}, \mathrm{U}$ & $253 \pm 10$ & $181 \pm 10$ & $\mathrm{~S} 11$ \\
\hline 60615 & $\mathrm{BD}+362268$ & 10.31 & B3V & $\mathrm{T}$ & $31 \pm 4$ & $54 \pm 4$ & S11 \\
\hline 61431 & HD 109399 & 7.67 & B0.5III & $\mathrm{F}$ & $-43 \pm 3$ & $203 \pm 6$ & $\mathrm{~T} 11$ \\
\hline 64458 & HD 114569 & 8.10 & B7 $/ 8$ & $\mathrm{~F}$ & $104 \pm 2$ & $74 \pm 1$ & M12 \\
\hline 67060 & HD 119608 & 7.53 & $\mathrm{~B} 1 \mathrm{Ib}$ & $\mathrm{F}$ & $31 \pm 1$ & $59 \pm 9$ & M04 \\
\hline 68297 & HD 121968 & 10.26 & $\mathrm{~B} 1 \mathrm{~V}$ & $\mathrm{~T}, \mathrm{U}$ & $17 \pm 9$ & $199 \pm 27$ & S11 \\
\hline 70205 & LP 857-24 & 11.36 & $\ldots$ & $\mathrm{F}$ & $243 \pm 4$ & $64 \pm 4$ & B12 \\
\hline 70275 & HD 125924 & 9.66 & B2IV & $\mathrm{T}$ & $244 \pm 1$ & $64 \pm 3$ & $\mathrm{~S} 11$ \\
\hline 79649 & HD 146813 & 9.06 & B 1.5 & $\mathrm{~T}$ & $21 \pm 2$ & $87 \pm 3$ & S11 \\
\hline 81153 & HD 149363 & 7.81 & B0.5III & $\mathrm{F}, \mathrm{T}, \mathrm{U}$ & $145 \pm 3$ & $88 \pm 10$ & $\mathrm{~S} 11$ \\
\hline 85729 & HD 158243 & 8.15 & $\mathrm{~B} 1 \mathrm{Ib}$ & $\mathrm{F}$ & $-63 \pm 2$ & $70 \pm 2$ & M12 \\
\hline 91049 & HD 171871 & 7.78 & B2IIp & $\mathrm{T}$ & $-64 \pm 1$ & $44 \pm 1$ & $\mathrm{~T} 11$ \\
\hline 92152 & HD 173502 & 9.70 & B1II & $\mathrm{F}$ & $49 \pm 1$ & $53 \pm 3$ & K82 \\
\hline 94407 & HD 179407 & 9.44 & $\mathrm{~B} 0.5 \mathrm{Ib}$ & $\mathrm{F}$ & $-120 \pm 4$ & $134 \pm 9$ & S97 \\
\hline 96130 & HD 183899 & 9.93 & B2III & $\mathrm{F}$ & $-46 \pm 2$ & $55 \pm 3$ & $\mathrm{~S} 11, \mathrm{~T} 11$ \\
\hline 98136 & HD 188618 & 9.38 & B2II & $\mathrm{F}$ & $46 \pm 4$ & $167 \pm 3$ & $\mathrm{~S} 11$ \\
\hline 101328 & HD 195455 & 9.20 & B0.5III & $\mathrm{F}, \mathrm{U}$ & $19 \pm 7$ & $213 \pm 7$ & $\mathrm{~S} 11$ \\
\hline 105912 & HD 204076 & 8.73 & $\mathrm{~B} 1 \mathrm{~V}$ & $\mathrm{~F}, \mathrm{U}$ & $0 \pm 3$ & $102 \pm 1$ & $\mathrm{~S} 11, \mathrm{~T} 11$ \\
\hline 107027 & HD 206144 & 9.34 & B2II & $\mathrm{F}, \mathrm{U}$ & $122 \pm 5$ & $184 \pm 12$ & $\mathrm{~S} 11$ \\
\hline 109051 & HD 209684 & 9.94 & B2/3III & $\mathrm{U}$ & $82 \pm 2$ & $108 \pm 3$ & $\mathrm{~S} 11, \mathrm{~T} 11$ \\
\hline 111563 & HD 214080 & 6.93 & B1/2Ib & $\mathrm{F}$ & $16 \pm 2$ & $108 \pm 3$ & $\mathrm{~S} 11$ \\
\hline 112022 & HD 214930 & 7.40 & B2IV & $\mathrm{T}$ & $-60 \pm 4$ & $12 \pm 1$ & M05 \\
\hline 112482 & HD 215733 & 7.34 & B1II & $\mathrm{T}$ & $-6 \pm 6$ & $72 \pm 1$ & $\mathrm{~T} 11$ \\
\hline 113735 & HD 217505 & 9.13 & B2III/IV & $\mathrm{F}$ & $-17 \pm 1$ & $26 \pm 2$ & S11 \\
\hline 114690 & HD 219188 & 7.06 & B0.5III & $\mathrm{F}, \mathrm{T}$ & $73 \pm 19$ & $239 \pm 15$ & $\mathrm{~S} 11$ \\
\hline 115347 & HD 220172 & 7.64 & $\mathrm{~B} 3 \mathrm{Vn}$ & $\mathrm{F}$ & $26 \pm 2$ & $39 \pm 1$ & $\mathrm{~S} 11$ \\
\hline 115729 & HD 220787 & 8.29 & B3III & $\mathrm{F}$ & $26 \pm 2$ & $26 \pm 2$ & $\mathrm{~S} 11, \mathrm{~T} 11$ \\
\hline$\cdots$ & EC 05582-5816 & 9.46 & B3V & $\mathrm{F}$ & $85 \pm 13$ & $221 \pm 5$ & S11 \\
\hline$\ldots$ & EC 13139-1851 & 10.50 & B4 & $\mathrm{U}$ & $15 \pm 4$ & $43 \pm 1$ & $\mathrm{~S} 11$ \\
\hline$\cdots$ & EC 20140-6935 & 8.83 & $\mathrm{~B} 2 \mathrm{~V}$ & $\mathrm{~F}$ & $-24 \pm 2$ & $45 \pm 2$ & $\mathrm{~S} 11$ \\
\hline$\cdots$ & PB 5418 & 11.35 & B2 & $\mathrm{U}$ & $147 \pm 3$ & $50 \pm 1$ & S11 \\
\hline$\ldots$ & PHL 159 & 10.90 & $\mathrm{~B}$ & $\mathrm{U}$ & $87 \pm 2$ & $30 \pm 1$ & $\mathrm{~S} 11$ \\
\hline
\end{tabular}

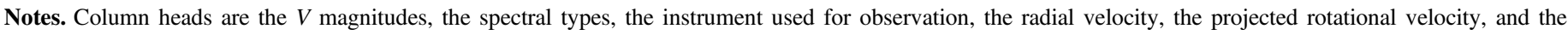
reference that identifies the star as a runaway.

a Spectrograph used for the observation: F-Feros, T-Tull, and U-UVES.

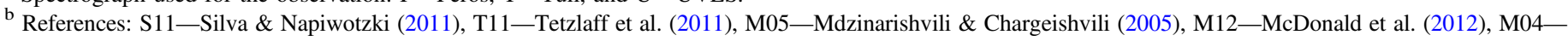
Martin (2004), B12_de Bruijne \& Eilers (2012), K82-Keenan et al. (1982), and S97-Smartt et al. (1997).

B stars of the same atmospheric parameters-see, for example, the runaway sample analyzed by Martin (2004). This is the first non-LTE analysis of a sample of runaway B stars.

In Section 2, we discuss the selection criteria used to isolate our sample of runaway stars whose spectra were obtained (see Section 3) and analyzed in Section 4 following the method previously applied by Hunter et al. (2009-see also Trundle et al. 2007) to B stars in the three Galactic open clusters. Abundances in the runaway and cluster B stars are discussed in Section 5. Rotational and radial velocities are discussed in Section 6. Brief concluding remarks are offered in Section 7.

\section{Selection of Runaway B Stars}

Targets were selected from catalogs of previously identified runaway candidates. Different criteria are outlined in each source identified in the reference column of Table 1 and cited in the footnotes to the table. The table lists the stars by their HIP number where available and/or an alternative designation, the $V$ magnitude, the spectral type, the source of our spectra (see the next section), the radial velocity $\left(v_{r}\right)$, the projected rotational velocity $(v \sin i)$, and the reference to the star's selection as a runaway B star. All objects in our sample either lie far from the Galactic plane with a height above or below the plane ( $z$ distance) of $>0.3 \mathrm{kpc}$ or have a high Galactic latitude $\left.|b|>30^{\circ}\right)$ or a peculiar space velocity of $\gtrsim 30 \mathrm{~km} \mathrm{~s}^{-1}$. A star that meets any one of these criteria is considered a runaway. Essentially each star in our sample has been shown by one or more of the references to have been ejected from the disk, i.e., the travel time from disk to its halo location is less than the lifetime of the star. In the BSS it is expected that the ejection 
velocity gained from the loss of mass from the binary system as a result of the SN will not exceed $300-400 \mathrm{~km} \mathrm{~s}^{-1}$, while for the CES similar velocities are possible (see Leonard 1993; Portegies Zwart 2000; Gvaramadze et al. 2009). A majority of the runaway stars are expected to be bound to the Galaxy. Stars exceeding the escape velocity are generally called "hypervelocity" stars, and the principal ejection engine for such stars is the Galaxy's central supermassive black hole (see review by Brown 2015). Two hypervelocity stars originating in the outer Galaxy are known: HD 271791 (Heber et al. 2008; Przybilla et al. 2008) and HIP 60350 (Irrgang et al. 2010). Such stars may be products of the BSS operating in a binary that has particular initial masses.

One of our two primary sources of runaway B stars is a list by Silva \& Napiwotzki (2011) of 174 high-Galactic-latitude B stars drawn from the literature. As noted by Silva \& Napiwotzki and emphasized by essentially all previous discussion of runaway B stars, the spectral classification of B type does not ensure that the star is a B main-sequence (massive) star because low-mass stars evolving either off the blue horizontal branch or from the asymptotic branch stars (i.e., post-AGB stars) can encroach on the effective temperaturesurface gravity $\left(T_{\mathrm{eff}}, \log g\right.$ ) plane occupied by main-sequence B stars-see also Tobin (1987). Using then-available information on $\left(T_{\text {eff }}, \log g\right)$ and data on atmospheric abundances, Silva \& Napiwotzki identified which of the 174 stars belong or possibly belong to the main sequence (MS or MS?). With one exception, the stars we observed from Silva \& Napiwotzki's Tables 2 and 3 were classified as MS or MS? The one exception was HIP 60615 (BD +36 2268), which was classified as non-MS. As massive stars, the combination of effective temperature and surface gravity indicate that the sample spans the mass range of about 5-25 solar masses. The estimated ejection velocities range up to about $400 \mathrm{~km} \mathrm{~s}^{-1}$ (Silva \& Napiwotzki 2011) and do not lead to escape from the Galaxy. The actual space velocities of observed runaways are smaller than the ejection velocities because stars are generally observed near the apex of their orbit where they spend most of their time.

Our second primary source of runaway B stars is the catalog of young runaway stars within $3 \mathrm{kpc}$ of the Sun compiled by Tetzlaff et al. (2011) using Hipparcos astrometry. We selected 13 B stars from this catalog of more than 2500 stars younger than about $50 \mathrm{My}$ and with peculiar velocities; of these, six also belong to our selection from Silva \& Napiwotzki (2011).

Our sample was completed by selecting another 10 targets from the literature on runaway stars.

\section{Observations}

High-resolution high signal-to-noise optical spectra were collected from three telescopes over a period of 16 months. The wavelength coverage and observation dates of each data set are shown in Table 2. Each set of observations is described below, along with the reduction procedures through which they were prepared for analysis.

\subsection{FEROS Observations}

Twenty-six targets were observed in 2014 August, using the ESO FEROS instrument (Kaufer et al. 1999), a high-resolution $(R \approx 48,000)$ prism cross-dispersed echelle spectrograph. FEROS has almost complete spectral coverage from 3500 to $9200 \AA$ and provides a high $\mathrm{S} / \mathrm{N}(\approx 300$ - see Table 2$)$ in
Table 2

Observation Dates and Wavelength Coverage of Each Instrument

\begin{tabular}{lll}
\hline \hline Instrument & $\lambda$ Coverage $(\AA)$ & Dates \\
\hline FEROS & $3500-9200$ & 2013 Aug \\
Tull & $3400-10900$ & 2013 May/Jun \\
UVES blue arm & $3730-4990$ & 2014 Mar-Jul \\
UVES red arm & $5650-9460$ & 2014 Mar-Jul \\
\hline
\end{tabular}

relatively short exposure times for bright stars. All data were reduced using the ESO FEROS pipeline (version 1.57). Multiple exposures were combined using either a median or a weighted average, within IRAF. ${ }^{6}$ Both these methods of merging the exposures resulted in very similar spectra, with the weighted average giving a slightly higher signal-to-noise ratio, and so these were adopted. Occasionally cosmic ray events were visible, but these were easily removed from the spectra manually, if they interfered with any analysis.

Four targets (HD 1999, HD 165955, HD 204076, and HD 208213) were deemed unusable for abundance analysis. HD 1999 showed double lines in its spectrum, making quantitative analysis unreliable. HD 165955 has a very high $v$ sin $i$, so many lines became unobservable. HD 204076 proved to be a doublelined spectroscopic binary when it was observed with UVES. HD 208213 did not have a sufficient $\mathrm{S} / \mathrm{N}$ to identify the Si III lines required for our analysis. It was also observed with UVES and discarded for the same reason.

\subsection{UVES Observations}

Seventeen targets were observed at the Very Large Telescope using the UVES instrument (Dekker et al. 2000), a high-resolution ( $R \approx 80,000)$, high-efficiency, cross-dispersed echelle spectrograph with a blue arm and a red arm. A standard setting $(437+760)$ was used, yielding a wavelength coverage of $3730-4990 \AA$ in the blue arm and 5650-9460 $\AA$ in the red arm. All of the data were taken directly from the ESO archive, having been reduced using the ESO UVES pipeline (Ballester et al. 2000). Multiple exposures were normalized and combined using either a median or a weighted $\sigma$-clipping algorithm, within IDL. As the $\mathrm{S} / \mathrm{N}$ of the individual exposures was high, the final spectra from both methods were effectively indistinguishable. Again, any cosmic ray events that interfered with subsequent analysis were removed manually.

As the UVES data set was obtained to extend our study to a higher $v \sin i$ and fainter targets, some of these proved particularly difficult to analyze and five were discarded, leaving 12 for which atmospheric parameters and abundances have been estimated.

\subsection{McDonald Observations}

Fourteen runaway candidates were observed at the W. J. McDonald Observatory with the Tull coudé echelle spectrograph at the $2.7 \mathrm{~m}$ Harlan Smith telescope (Tull et al. 1995), with a high spectral resolution $(R \approx 60,000)$, during 2013 May and June. Spectra were obtained covering a wavelength range of 3400-10900 ̊. The echelle data were

\footnotetext{
6 IRAF is distributed by the National Optical Astronomy Observatory, which is operated by the Association of Universities for Research in Astronomy under cooperative agreement with the National Science Foundation.
} 
split into orders, each of which was reduced separately using standard IRAF procedures.

For stars with significant rotational broadening and/or large surface gravities, the $\mathrm{H} \delta$ line profiles extended over a significant fraction of the order. Therefore, the normalization of these data proved more difficult than the normalization of those from UVES and FEROS. To deal with this concern, blaze fits were made to the bracketing orders, where the continuum was obvious, and these were averaged to provide a blaze profile for the order containing $\mathrm{H} \delta$. This was then used to rectify the orders containing $\mathrm{H} \delta$. All data were subsequently normalized and multiple exposures combined using either a median or a weighted $\sigma$-clipping algorithm, within IDL, as with the UVES observations discussed above.

Three of the McDonald targets were not analyzed. HD 69686 and HD 118246 did not have a sufficient S/N to identify the rotationally broadened $\mathrm{Si}$ III lines, preventing estimates of the projected rotational velocities, effective temperatures, and microturbulences. HD 203664 had complex and variable spectra (Aerts et al. 2006) and so was removed from the sample.

\section{Method of Analysis}

Non-LTE model atmosphere grids and model atoms from the TLUSTY and SYNSPEC codes (Hubeny 1988; Hubeny \& Lanz 1995; Hubeny et al. 1998; Lanz \& Hubeny 2007) were used to derive atmospheric parameters and chemical abundances. More detailed discussions of our analysis methods can be found in Hunter et al. (2007), while those of the atmospheric grids are in Ryans et al. (2003) and Dufton et al. (2005). ${ }^{7}$ Hence only a brief summary is given here.

Model atmosphere grids have been generated with metallicities representative of the Galaxy $([\log (\mathrm{Fe} / \mathrm{H})+12]=7.5 \mathrm{dex}$, and other abundances scaled accordingly). These model atmospheres cover a range of effective temperatures from 12,000 to $35,000 \mathrm{~K}$ in steps of 500-1500 K, and surface gravities ranging from close to the Eddington limit to $4.5 \mathrm{dex}$ in steps of $0.15 \mathrm{dex}$ (Hunter et al. 2008).

The codes make non-LTE assumptions, i.e., the atmospheres can be considered plane parallel with winds having no significant effect on the optical spectrum, and a normal $\mathrm{He}-\mathrm{H}$ ratio ( 0.1 by number of atoms) is assumed. Dufton et al. (2005) and McEvoy et al. (2015) independently tested the validity of this approach. They analyzed the spectra of B-type supergiants in the SMC and the $\mathrm{LMC}$, respectively, using the grids described here and also the FASTWIND code (Santolaya-Rey et al. 1997; Puls et al. 2005), which incorporates wind effects. Dufton et al. (2005) found excellent agreement in the atmospheric parameters estimated from the two methods. The effective temperature, logarithmic surface gravity, and microturbulent velocity estimates all agreed to well within their errors. The abundance values agreed to within 0.1 dex for elements such as $\mathrm{C}, \mathrm{O}, \mathrm{Si}$, and $\mathrm{Mg}$, while the discrepancies in $\mathrm{N}$ abundances were less than $0.2 \mathrm{dex}$, although a systematic difference of $\sim 0.1$ dex did appear to exist between the two approaches. Dufton et al. (2005) suggested that this was due to differences in the $\mathrm{N}$ model atoms and the wind effects adopted. McEvoy et al. (2015) also found good agreement between results from the two codes. Of the 11 stars analyzed using both methods, five targets had effectively identical results and five

\footnotetext{
See also http://star.pst.qub.ac.uk.
}

agreed well (with differences $\leqslant 1000 \mathrm{~K}$ in $T_{\text {eff }}, \leqslant 0.1 \mathrm{dex}$ in $\log g$, and $\leqslant 0.2 \operatorname{dex}$ in $\mathrm{N}$ abundance estimates), with the $\mathrm{N}$ abundance in only one target showing significant discrepancies between the methods. However, this star is an extreme object, close to the Eddington limit, where $\mathrm{N}$ abundances will be less secure. As the majority of our sample is lower-luminosity dwarfs or giants, our approach should be adequate.

We adopt baseline chemical abundances from Hunter et al. (2007, 2009) derived from a sample of B-type stars in the Milky Way. Other B-type stellar studies, such as those by Lyubimkov et al. (2005, 2013), Daflon et al. (2009), SimónDíaz et al. (2010), and Nieva \& Simón-Díaz (2011), have found higher baseline abundances for $\mathrm{Mg}, \mathrm{Si}$, and $\mathrm{N}$, closer to Solar abundances, but to maintain consistency in our analysis we use the Hunter baseline values that for $\mathrm{N}, \mathrm{Si}$, and $\mathrm{Mg}$ are 7.62, 7.42, and 7.25 dex, respectively (see Hunter et al. 2007, 2009).

It is important to note that the stellar metallicity distribution in a disk galaxy, including the Milky Way, typically exhibits a negative gradient as a function of distance from the Galactic center, in both the radial and the vertical directions (Huang et al. 2015). Examples of studies where radial $\mathrm{N}$ abundance gradients in the Galactic disk have been studied include Rolleston et al. (2000), who found a gradient of $-0.09 \pm 0.01 \mathrm{dex} \mathrm{kpc}-1$, while Daflon \& Cunha (2004) found an average gradient for all elements in the Galactic disk of $-0.042 \pm 0.007 \mathrm{dex} \mathrm{kpc}{ }^{-1}$, with an $\mathrm{N}$ value of $-0.046 \pm 0.011 \mathrm{dex} \mathrm{kpc}^{-1}$, half of that estimated by Rolleston et al. (2000). Both of these measurements rely on abundances in young OB-type stars. Shaver et al. (1983) used radio and optical spectroscopy to sample Galactic H II regions, spanning 3.5-13.7 kpc from the Galactic center. They found a $\mathrm{N}$ abundance gradient of $-0.09 \pm 0.015 \mathrm{dex} \mathrm{kpc}{ }^{-1}$, similar to Rolleston et al. (2000), along with evidence of steeper gradients in the inner regions of the Galactic disk. Huang et al. (2015) investigated this effect both radially and vertically using 7000 red clump stars between 7 and $14 \mathrm{kpc}$ from the Galactic center. They found that between 7 and $11.5 \mathrm{kpc}$ the radial gradient flattens as the height from the Galactic plane increases, but that between 11.5 and $14 \mathrm{kpc}$ the gradients do not vary with height and are at a constant value of $-0.014 \mathrm{dex} \mathrm{kpc}^{-1}$. Rolleston et al. (2000) also considered a two-zone model but found no evidence to indicate that this was more appropriate. Here, an average $\mathrm{N}$ abundance for Galactic B-type stars has been adopted from Hunter et al. (2009), although this value may be slightly higher if stars were formed closer to the Galactic center and lower if they were formed far from it. However, in no case has the gradient been found to be very large, so the metallicity distribution should not have a significant effect on the results presented here.

\subsection{Atmospheric Parameters}

The three characteristic parameters of a static stellar atmosphere (effective temperature, surface gravity, and microturbulence) are interdependent, so an iterative process was used to estimate these values (see Fraser et al. 2010; McEvoy et al. 2015 for more details). The parameters are described separately in the subsections below. The final values for these parameters are given in Table 3.

\subsubsection{Effective Temperature}

Effective temperature ( $T_{\text {eff }}$ ) estimates were determined using the $\mathrm{Si}$ ionization balance. Equivalent widths of the $\mathrm{Si}$ III 
Table 3

Final Atmospheric Parameters for Each Star, Listed by HIP Number, with Another Identifier Shown ${ }^{\mathrm{a}}$

\begin{tabular}{|c|c|c|c|c|c|c|c|c|}
\hline HIP & Other & $\underset{\mathrm{K}}{T_{\text {eff }}}$ & $\operatorname{cog~s}^{\log g}$ & $\begin{array}{c}v_{\mathrm{t}} \\
\mathrm{km} \mathrm{s}^{-1}\end{array}$ & $\mathrm{Mg}$ & $\mathrm{Si}$ & $\mathrm{N}$ & $\mathrm{C}$ \\
\hline 2702 & HD 3175 & 16100 & 3.6 & 8 & 6.85 & 7.02 & 7.35 & 7.82 \\
\hline 3812 & CD -56 152 & 17000 & 3.4 & 14 & 6.83 & 6.83 & 7.42 & $\cdots$ \\
\hline 7873 & HD 10747 & 18700 & 3.8 & 8 & 7.03 & 7.01 & 7.33 & 7.69 \\
\hline 13489 & HD 18100 & 23500 & 3.6 & 15 & 7.05 & 6.64 & 7.42 & $\cdots$ \\
\hline 16758 & HD 22586 & 21700 & 3.3 & 14 & 7.44 & 7.62 & 7.93 & 8.13 \\
\hline 45563 & HD 78584 & 18600 & 3.8 & 8 & 7.01 & 6.81 & 7.31 & $\ldots$ \\
\hline 55051 & HD 97991 & 21500 & 3.8 & 5 & 6.98 & 7.36 & 7.58 & $\ldots$ \\
\hline 56322 & HD 100340 & 24500 & 3.8 & 4 & 7.43 & 7.55 & 7.61 & $\ldots$ \\
\hline 60615 & $\mathrm{BD}+362268$ & 19600 & 3.4 & 9 & 6.97 & 6.82 & 7.66 & $\cdots$ \\
\hline 61431 & HD 109399 & 23000 & 3.1 & 16 & 7.38 & 7.24 & 7.42 & $\cdots$ \\
\hline 64458 & HD 114569 & 18300 & 3.8 & 6 & 7.21 & 7.24 & 7.90 & 8.39 \\
\hline 67060 & HD 119608 & 19900 & 2.7 & 16 & 7.52 & 7.67 & 7.93 & 8.05 \\
\hline 68297 & HD 121968 & 20550 & 3.4 & 0 & 7.43 & 7.98 & 7.72 & $\cdots$ \\
\hline 70205 & LP 857-24 & 24600 & 4.1 & 0 & 7.45 & 7.46 & 7.42 & $\cdots$ \\
\hline 70275 & HD 125924 & 21000 & 3.6 & 6 & 7.08 & 7.13 & 7.28 & $\cdots$ \\
\hline 79649 & HD 146813 & 19400 & 3.2 & 5 & 7.02 & 7.25 & 7.47 & $\cdots$ \\
\hline 81153 & HD 149363 & 27800 & 3.5 & 12 & 7.60 & 7.79 & 7.86 & 8.22 \\
\hline 85729 & HD 158243 & 19300 & 2.7 & 20 & 7.37 & 7.69 & 7.91 & 8.02 \\
\hline 91049 & HD 171871 & 20300 & 3.4 & 14 & 7.35 & 7.51 & 7.81 & $\cdots$ \\
\hline 92152 & HD 173502 & 25600 & 3.5 & 12 & 7.51 & 7.74 & 7.98 & $\ldots$ \\
\hline 94407 & HD 179407 & 26000 & 3.4 & 17 & 8.00 & 7.82 & 8.21 & 8.53 \\
\hline 96130 & HD 183899 & 20000 & 3.3 & 17 & 7.18 & 7.53 & 7.80 & 7.98 \\
\hline 98136 & HD 188618 & 21300 & 3.4 & 11 & 7.32 & 7.34 & 7.85 & $\ldots$ \\
\hline 101328 & HD 195455 & 20550 & 3.2 & 14 & 7.47 & 7.74 & 7.96 & 7.83 \\
\hline 105912 & HD 204076 & 20100 & 3.4 & 20 & 7.19 & 7.43 & 7.97 & $\cdots$ \\
\hline 107027 & HD 206144 & 17750 & 2.5 & 16 & 7.24 & 7.31 & 7.56 & 7.68 \\
\hline 109051 & HD 209684 & 20340 & 3.9 & 9 & 6.98 & 7.17 & 7.54 & $\cdots$ \\
\hline 111563 & HD 214080 & 19400 & 2.9 & 17 & 7.18 & 7.63 & 7.56 & 7.81 \\
\hline 112022 & HD 214930 & 18000 & 3.4 & 6 & 7.04 & 7.04 & 7.27 & $\ldots$ \\
\hline 112482 & HD 215733 & 23100 & 2.9 & 14 & 7.39 & 7.40 & 7.60 & $\cdots$ \\
\hline 113735 & HD 217505 & 21600 & 3.9 & 3 & 7.27 & 7.33 & 7.56 & 7.97 \\
\hline 114690 & HD 219188 & 23200 & 3.0 & 13 & 7.37 & 7.71 & 7.66 & 7.86 \\
\hline 115347 & HD 220172 & 21700 & 3.8 & 0 & 7.31 & 7.41 & 7.69 & 7.95 \\
\hline 115729 & HD 220787 & 18600 & 3.6 & 5 & 7.01 & 7.07 & 7.51 & 7.91 \\
\hline$\cdots$ & EC 05582-5816 & 15900 & 3.4 & 10 & 6.97 & 7.43 & 8.40 & 8.39 \\
\hline$\cdots$ & EC 13139-1851 & 18100 & 3.9 & 13 & 7.09 & 7.21 & 7.64 & $\cdots$ \\
\hline$\cdots$ & EC 20140-6935 & 21900 & 3.8 & 0 & 7.20 & 7.47 & 7.72 & 8.09 \\
\hline$\cdots$ & PB 5418 & 19300 & 3.8 & 8 & 7.05 & 7.07 & 7.44 & $\cdots$ \\
\hline$\cdots$ & PHL 159 & 22900 & 4.1 & 0 & 7.46 & 7.41 & 7.46 & $\cdots$ \\
\hline
\end{tabular}

Notes. The effective temperatures, surface gravities, and microturbulences are given for each star, along with the $\mathrm{Mg}, \mathrm{Si}, \mathrm{N}$, and $\mathrm{C}$ abundances where available.

${ }^{a}$ Analysis of EC 05582-5816 is based on model atmospheres with the Si abundance set to 7.4. For all other analyses, the Si abundance is determined from Si lines and the microturbulence is determined from the Si III lines.

multiplet $(4552,4567,4574 \AA$ ) were measured, together with those for the Si IV lines at 4089 and $4116 \AA$ in the hotter targets and those of $\mathrm{Si}$ II at $4128,4130 \AA$ in the cooler stars. For narrow-lined, high $\mathrm{S} / \mathrm{N}$ targets, a simple Gaussian profile fit was sufficient to obtain a reliable equivalent width measurement (see Figure 1, upper left plot). The uncertainties in these measurements are typically of the order of $10 \%$ (Hunter et al. 2007). For stars with higher projected rotational velocities $\left(v \sin i \geqslant 50 \mathrm{~km} \mathrm{~s}^{-1}\right)$, it was more appropriate to fit rotationally broadened profiles, as rotation becomes the dominant broadening mechanism (see Figure 1, upper right plot). In some cases it was not possible to measure the strength of either the Si II or the Si IV spectrum. For these targets upper limits were set on their equivalent widths, allowing constraints to the effective temperatures to be estimated (see, e.g., Hunter et al. 2007 for more details). This was mostly the case for stars with large projected rotational velocities and midrange temperatures $(18,000-26,000 \mathrm{~K})$. The random uncertainty in our effective temperatures is approximately $\pm 1000 \mathrm{~K}$ (about $5 \%$ ), consistent with the high quality of the observational data. In those cases where upper limits were set for the equivalent widths of the Si absorption lines, the values will obviously be more uncertain and so error bars of $\approx 2000 \mathrm{~K}$ are more appropriate.

\subsubsection{Surface Gravity}

The logarithmic surface gravity $(\log g)$ was estimated by comparing the theoretical and the observed profiles of the hydrogen Balmer line $\mathrm{H} \delta$. Automated procedures were developed to fit the theoretical spectra to the observed lines, with regions of best fit defined using contour maps of $\log g$ against $T_{\text {eff. }}$ Using the effective temperatures deduced by the methods outlined above, the gravity could be estimated. The effects of instrumental, rotational, and macroturbulent broadening, which have the most significant effect on the line cores, 

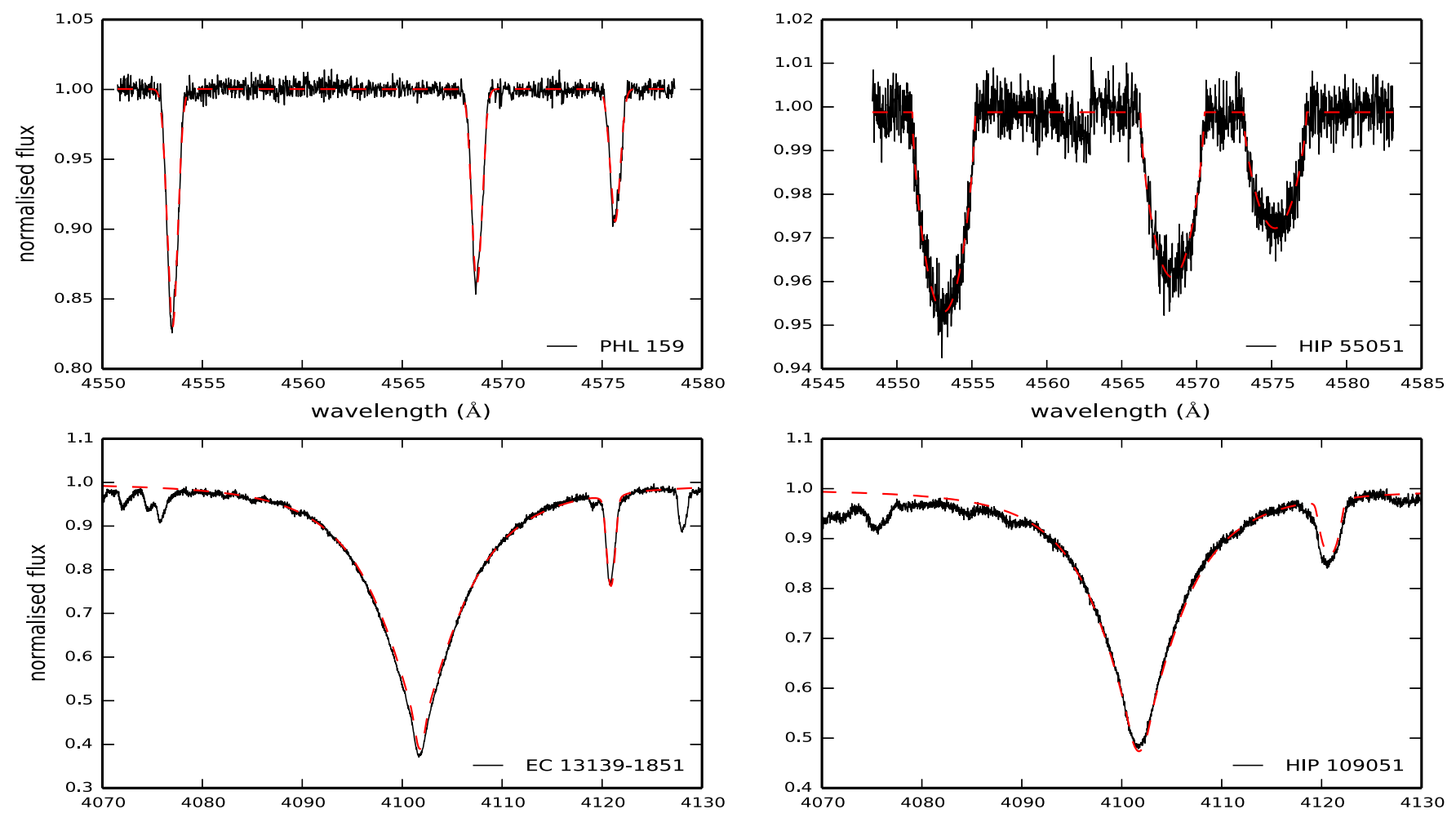

Figure 1. Upper left plot: Si III spectrum for PHL $159\left(v \sin i=30 \mathrm{~km} \mathrm{~s}^{-1}\right)$, with Gaussian model fits (red dotted line) used to determine $v_{\mathrm{t}}$ and $T_{\text {eff. }}$ Upper right plot: $\mathrm{Si}$ III spectrum for HIP $55051\left(v \sin i=135 \mathrm{~km} \mathrm{~s}^{-1}\right)$, with rotationally broadened model fits (red dotted line) used to determine $v_{\mathrm{t}}$ and $T_{\text {eff. }}$ Lower left plot: $\mathrm{H} \delta$ spectrum for EC 13139-1851 $\left(v \sin i=43 \mathrm{~km} \mathrm{~s}^{-1}\right.$ ), along with its model fit, used to determine $\log g$. Lower right plot: $\mathrm{H} \delta$ spectrum for HIP 109051 $\left(v \sin i=108 \mathrm{~km} \mathrm{~s}^{-1}\right)$, along with its model fit, used to determine $\log g$.

were considered in the theoretical profiles (see Figure 1, lower plots). Uncertainties in the fitting procedures led to random errors of \pm 0.1 dex, while systematic errors could be present due to, e.g., the uncertainty in the adopted line broadening theory or in the model atmosphere assumptions. Additional errors might arise from the uncertainty of the identification of the continuum around $\mathrm{H} \delta$ in the McDonald spectra (see Section 3.3), where less continuum was available around the line.

\subsubsection{Microturbulence}

Following standard practice, we derived the microturbulent velocity from the Si III triplet $(4552,4567$, and $4574 \AA$ - see upper plots in Figure 1) as it is observed in all our analyzable spectra and because all three lines arise from the same multiplet, so that errors in the absolute oscillator strengths and non-LTE effects should be minimized.

An alternative approach to the microturbulence determination uses the same Si III triplet but finds the microturbulence that gives the baseline Si abundance of 7.42 from Hunter et al. (2009) as the average from the three lines. This alternative also impacts the determination of the effective temperature and surface gravity and hence the abundances of all elements. In the next section, we comment on the effect on the abundances of the two methods for determining the microturbulence.

\subsection{Elemental Abundances}

$\mathrm{N}, \mathrm{Mg}, \mathrm{Si}$, and in some cases $\mathrm{C}$ abundances for each star have been estimated using the atmospheric parameters given in Table 3 and measurements of the absorption lines. Abundances are given in Table 3. The atmospheric parameters agree well between the observations with the different spectrographs. Effective temperatures show differences not larger than $1200 \mathrm{~K}$ in all cases, while the range of the logarithmic gravity estimates is only greater than 0.2 dex in one instance (HD 121968 with a range of $0.25 \mathrm{dex}$ ). Differences in microturbulence are less than $6 \mathrm{~km} \mathrm{~s}^{-1}$ in all cases. The agreement between atmospheric parameters derived from observations obtained with different instruments is reassuring but not surprising given the high quality of all spectra. This agreement is of course not a measure of any systematic errors.

Extensive appendices in Hunter et al. (2007) show how errors in the atmospheric parameters for B-type stars translate into errors in derived abundances. Hunter et al. (2007) considered the errors to be independent but in reality the situation will be more complicated, as this is not the case. For example, an increase in the effective temperature estimate will lead to an increase in the gravity estimate, and this leads to the theoretical $\mathrm{N}$ II equivalent widths (and hence $\mathrm{N}$ abundances) being less sensitive to changes in the atmospheric parameters than if these are considered to vary independently.

For the range in atmospheric parameters found for our sample, the simulations of Hunter et al. (2007; see, e.g., their Figure 6 for the N II $3995 \AA$ line) imply that our estimated errors in both the gravity and the microturbulence lead to relatively small errors in the $\mathrm{N}$ abundance estimate of $0.1 \mathrm{dex}$ or less. Errors in effective temperature estimates are more important and lead to larger uncertainties with an error of $\Delta T_{\text {eff }} \pm 1000 \mathrm{~K}$ translating into an $\mathrm{N}$ abundance error of approximately $\mp 0.2 \mathrm{dex}$ at an effective temperature of $18,000 \mathrm{~K}$ but decreasing to $\sim 0.1 \mathrm{dex}$ at an effective temperature of $25,000 \mathrm{~K}$. In addition, there will be random errors in the $\mathrm{N}$ abundance estimates due to uncertainties in the $\mathrm{N}$ II 


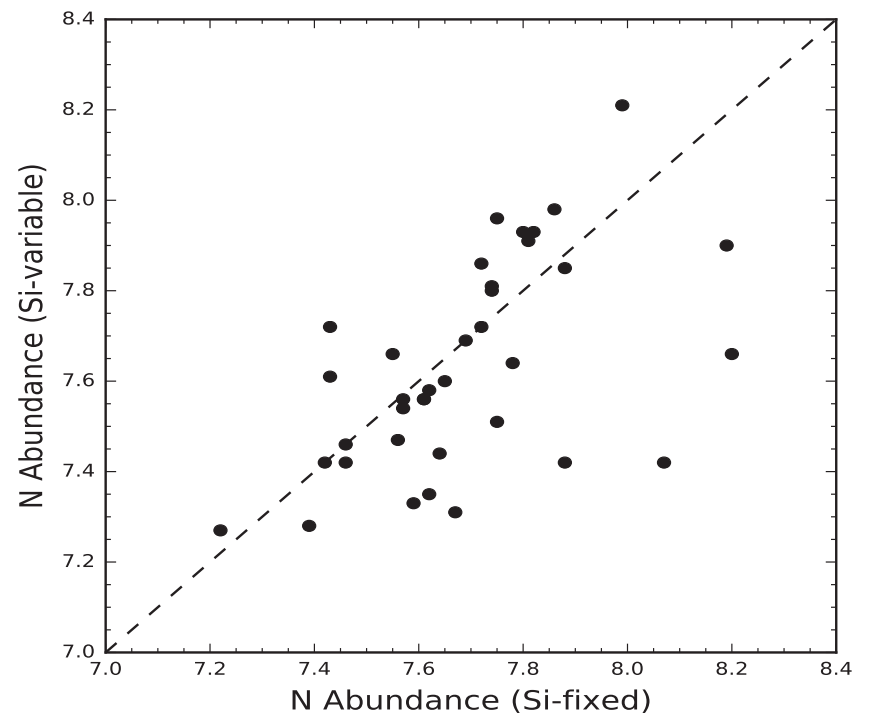

Figure 2. $\mathrm{N}$ abundances from models with the microturbulence set by the condition that the $\mathrm{Si}$ abundance is 7.40 vs. the $\mathrm{N}$ abundances set by the determination of the microturbulence from the Si III lines. The solid line of unit slope corresponds to an abundance independent of the method of fixing the microturbulence.

equivalent widths. The latter have been estimated as $\pm 10 \%$, which would imply an uncertainty of $\sim 0.1$ dex. Combining these different sources of error in quadrature would lead to typical uncertainties of $0.2-0.3$ dex.

For the atmospheric parameters and abundances in Table 3, the microturbulence was derived from the three Si III lines with the condition that the three lines return the same $\mathrm{Si}^{2+}$ abundance. This method was successful for all but EC 05582-5816, for which lines are greatly rotationally broadened. As we noted above, the alternative method is to assume a standard $\mathrm{Si}$ abundance of 7.4 for the determination of the microturbulence and hence other atmospheric parameters that finally define the model atmosphere used to determine the abundances of $\mathrm{C}, \mathrm{N}$, and $\mathrm{Mg}$. (This alternative method was used by Hunter et al. 2007 in their analysis of B stars in three Galactic clusters.) The $\mathrm{C}, \mathrm{N}$, and $\mathrm{Mg}$ abundances are little affected by how the microturbulence is chosen; the preferred and alternative methods give similar results. This is well shown by Figure 2, where we show the $\mathrm{N}$ abundances obtained when the microturbulence is set by the condition that the $\mathrm{Si}$ abundance is 7.4 plotted versus the abundances obtained when the microturbulence comes from the three Si III lines. It is seen that the $\mathrm{N}$ abundances are not systematically different in the two cases except for two or three outliers. Obvious outliers are HD 18100 and BD +36 2268 with $\mathrm{N}$ abundances obtained from the alternative method of 7.97 and 8.20, respectively. A similar correspondence between abundances from the two methods is found for $\mathrm{Mg}$ and for the limited number of determinations of the $\mathrm{C}$ abundances. Thus, apart from the artificial constraint of a constant $\mathrm{Si}$ abundance, the abundance analyses for all other elements are expected to be largely unaffected by the method used to determine the microturbulence and hence the atmospheric parameters.

For a subsample of our stars (all stars observed with FEROS) we estimated C abundances. The C II line at $4267 \AA$ was used to calculate abundances as it is the only measurable line in all of the spectra. This line is known to be susceptible to (subtle) non-LTE effects (Nieva \& Przybilla 2006, 2008), which are not fully taken

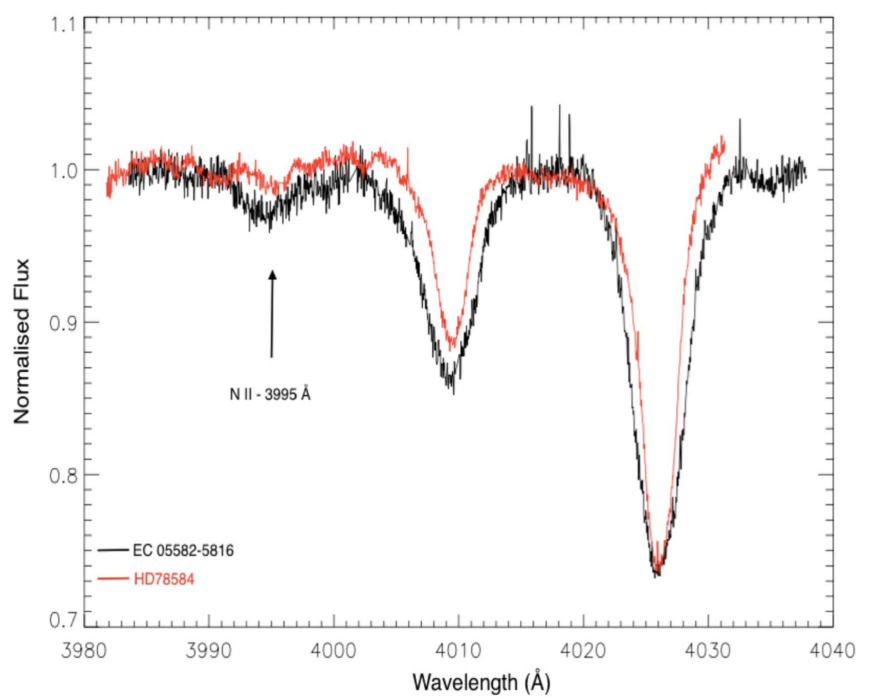

Figure 3. Spectrum around the N II line at $3995 \AA$ for the most N-enhanced star, EC 05582-5816. HD 78584 is overplotted in red for comparison, as it has a similar spectral type (B3) and a $v \sin i$ of $102 \mathrm{~km} \mathrm{~s}^{-1}$. Although the $\mathrm{N}$ line is heavily broadened in EC 05582-5816, due to its very high $v \sin i$ of $221 \mathrm{~km} \mathrm{~s}^{-1}$, it can still clearly be seen so it must be particularly strong to avoid being smeared out into the surrounding continuum.

into account in the model ion that was included in the TLUSTY calculations-see discussion by Hunter et al. (2009). Our interpretation of the various abundances is in part referenced to the abundance analysis of Galactic clusters by Hunter et al. (2007), who also exclusively used the $4267 \AA$ line; thus the inability to account fully for the non-LTE effects should be almost canceled by the comparison with the Galactic clusters.

$\mathrm{N}$ abundances in Table 3 were estimated from the singlet transition at $3995 \AA$ as it is one of the strongest $\mathrm{N}$ II lines in the optical spectrum and appears unblended. Two examples of spectra of high $v \sin i$ stars around the $\mathrm{N}$ line at $3995 \AA$ are shown in Figure 3. Other $\mathrm{N}$ II lines were also present in a significant number of observations, including those between 4601 and $4643 \AA$ and the singlet at $4447 \AA$. These tended to be more blended and so may lead to less accurate abundance estimates than those from $3995 \AA$. It is interesting to note that Lyubimkov et al. (2013) found that the line at $3995 \AA$ gave a lower $\mathrm{N}$ abundance in their sample compared with the abundance from other transitions and that the differences were a function of effective temperature, ranging from $-0.3 \mathrm{dex}$ at $16,000 \mathrm{~K}$ to $0.0 \mathrm{dex}$ at $29,000 \mathrm{~K}$. In our case, the difference is about -0.1 dex over the full temperature range with a rise to 0.0 dex below about $18,000 \mathrm{~K}$. These differences with Lyubimkov et al. (2013) likely reflect differences in the adopted model atoms. By referencing our abundances from the $3995 \AA$ line to those from Hunter et al. (2009), we expect to obtain a true measure of abundance differences between the cluster (i.e., the Galactic disk) and the runaway B stars. $\mathrm{Mg}$ abundances were estimated for all spectra using the $\mathrm{Mg}$ II transition at $4481 \AA$, comprising three overlapping lines of a single multiplet.

$\mathrm{Si}$ abundances are obtained from the lines of Si II, Si III, and Si IV referred to in the determination of the atmospheric parameters.

\section{Ejection Mechanisms and Abundances}

The most satisfying discussion of the abundances would conclude with the demonstration that runaway B stars formed 
by the BSS and the CES have distinct differences in composition with both scenarios providing abundance differences with B stars in Galactic open clusters. Perhaps the least satisfying result would be one in which the runaway B stars showed a common pattern of abundances and one that matched well the abundances of B stars in Galactic open clusters-i.e., the BSS and CES scenarios both preserve the abundances of the investigated elements. Yet in this case one hopes that the origin of runaway stars may be traced from other observational indicators such as binarity and rotational velocities.

The X-ray sources known as low-mass X-ray binaries (LMXBs) consist of a low-mass "normal" secondary star orbiting a $\mathrm{BH}$ or an NS where the $\mathrm{SN}$ leading to the $\mathrm{BH}$ or NS possibly contaminated the secondary. Abundance analysis of the secondary should offer clues to the contamination expected in the BSS mode of runaway star formation. One LMXB secondary accompanying a BH has a spectral type of B9III and with an effective temperature of $10,500 \mathrm{~K}$ approximates a runaway star. With $[\mathrm{Fe} / \mathrm{H}]=0.0$, this secondary in V4641 Sgr has a normal composition $(\mathrm{C}, \mathrm{O}, \mathrm{Mg}, \mathrm{Al}, \mathrm{Si}$, and $\mathrm{Ti}$ ) but an +0.8 dex overabundance for $\mathrm{N}$ and $\mathrm{Na}$ (Sadakane et al. 2006). Curiously, this star appears to be a replica of EC 05582-5816 (see Section 5.3). Unfortunately $\mathrm{C}$ and $\mathrm{N}$ abundances have not been reported for the other six LMXB secondaries. The secondary with the most extreme enrichments would appear to be Nova Sco 1994 (González Hernández et al. 2008) with, for example, $[\mathrm{Fe} / \mathrm{H}]=-0.1$ but $[\mathrm{Mg} / \mathrm{Fe}]=+0.4$ and $[\mathrm{Si} / \mathrm{Fe}]=+0.7$ and other anomalies including $[\mathrm{O} / \mathrm{Fe}]=+1.0$ and $[\mathrm{S} / \mathrm{Fe}]=+0.9$. $\mathrm{Mg}$ and $\mathrm{Si}$ abundance estimates are available for five secondaries. Comparisons with normal F, G, and $\mathrm{K}$ dwarfs are made in the $[\mathrm{Fe} / \mathrm{H}]$ versus $[\mathrm{X} / \mathrm{Fe}]$ plane where $\mathrm{X}=\mathrm{Mg}$ or $\mathrm{Si}$. The mean $[\mathrm{Fe} / \mathrm{H}]$ for the five stars is +0.1 with a spread from -0.1 to +0.3 . The mean difference with respect to normal stars of the same $[\mathrm{Fe} / \mathrm{H}]$ is $[\mathrm{Mg} / \mathrm{Fe}]$ and $[\mathrm{Si} / \mathrm{Fe}]$ at +0.2 with a spread from 0.0 to about +0.7 , but the $\mathrm{Mg}$ and $\mathrm{Si}$ enrichments would be larger if $\mathrm{Fe}$ were also enriched. There is a hint that $[\mathrm{Mg} / \mathrm{Fe}]$ and $[\mathrm{Si} / \mathrm{Fe}]$ are positively correlated. Other elements considered for four or more of the secondaries but not included in our analysis include the following with mean differences of $[\mathrm{X} / \mathrm{Fe}]$ relative to normal stars: $+0.4(\mathrm{O}),+0.4(\mathrm{Na}),+0.1(\mathrm{Al}), 0.0(\mathrm{Ca})$, +0.2 (Ti), and +0.1 (Ni). Again, elemental abundance enrichments are larger if SN Fe contaminates the secondary. Predicted enrichments depend on many factors including uncertain aspects of the SN explosion and do not completely account for the composition of these secondaries. In summary, as far as the elements considered here are concerned the observations of $\mathrm{C}$ and $\mathrm{N}$ are too few to suggest a pattern but $\mathrm{Mg}$ and $\mathrm{Si}$ may be enriched simultaneously in some cases.

Possible changes of composition occurring at the birth of a runaway star have to be extracted from the observed composition, bearing in mind that two other factors may affect the chemical composition of the B stars prior to their conversion to a runaway star: first, the initial composition of $\mathrm{B}$ (and other) stars depends on the location of their birth site; studies of the composition of stars and H II regions show that abundances decline with increasing distance from the Galactic center. For example, Daflon \& Cunha (2004) (regarding B stars) and Luck \& Lambert (2011) (regarding Cepheids) find that the abundance gradients for our elements are about $-0.045 \mathrm{dex} \mathrm{kpc}^{-1}$, a value representative of many other studies. Azimuthal variations are considered to be smaller than the radial variation. Since runaway stars in the sample catalogs are expected to have birth sites spanning several $\mathrm{kpc}$ in the Galactic disk, abundance variations of several 0.1 dex may be anticipated. Second, B stars may be prone to mixing between the surface and the interior, resulting in changes of composition as $\mathrm{CN}$-cycled $\mathrm{H}$-burning products reach the atmosphere-i.e., $\mathrm{He} / \mathrm{H}$ is increased with a coupled decrease of $\mathrm{C}$ and an increase of the $\mathrm{N}$ abundance-see, for example, calculations of surface abundances along evolutionary tracks for rotating massive stars by Brott et al. (2011). (Extreme mixing may contaminate the atmosphere with $\mathrm{ON}$-cycled $\mathrm{H}$-burning products, resulting in decreases of $\mathrm{O}$ and additional increases of $\mathrm{N}$ and $\mathrm{He}$.)

\section{1. $\mathrm{Mg}$ and $\mathrm{Si}$}

Since the $\mathrm{Mg}$ and $\mathrm{Si}$ abundance should be unaffected by mixing within evolving B stars but could conceivably be altered in runaway stars created by the BSS, we discuss this pair of elements ahead of our discussion of $\mathrm{C}$ and N. Our reference Galactic B stars are the three open clusters analyzed by Hunter et al. (2009): NGC 6611, at a Galactocentric distance $R_{G}$ of $6.1 \mathrm{kpc}$; NGC 3293 , at $R_{G}=7.6 \mathrm{kpc}$; and NGC 4755 , at $R_{G}=8.2 \mathrm{kpc}$, where $R_{G}$ is taken from Rolleston et al. (2000). (Daflon \& Cunha 2004 give somewhat different estimates.) Abundances provided by Hunter et al. (2009) correspond to the atmospheric parameters chosen by assuming an $\mathrm{Si}$ abundance of 7.4 (see Section 4 for comments on such a choice). Since the $2.1 \mathrm{kpc}$ difference in Galactocentric distance across the three open clusters should correspond to an abundance difference of only about $0.09 \mathrm{dex}$, the assumption of constant $\mathrm{Si}$ abundance is likely not a source of significant systematic error. The $\mathrm{Mg}$ abundances for the three Galactic clusters are just consistent with the anticipated 0.09 dex decline between NGC 6611 and NGC 4755. Of course the Si abundances are not expected to betray the abundance gradient because the condition $\mathrm{Si}=7.4$ was imposed on the analysis.

For the runaway $\mathrm{B}$ stars, the $\mathrm{Mg}$ and $\mathrm{Si}$ abundances from Table 3 are compared in Figure 4, which shows that $\mathrm{Mg}$ and $\mathrm{Si}$ abundances are highly correlated and that each range over 1.0 dex. The straight line in Figure 4 corresponds to a constant $\mathrm{Mg} / \mathrm{Si}$ ratio set at the "standard" ratio of -0.17 dex adopted by Hunter et al. (2009). The spread in Mg abundances among the runaway stars far exceeds the range among stars from an individual open cluster, which is a fair measure of the measurement uncertainties given that stars within a cluster share a common $\mathrm{Mg}$ abundance. A major contribution to the $\mathrm{Mg}$ and $\mathrm{Si}$ abundance spread among the runaway stars surely arises because their birthplaces span quite a range in Galactocentric distances even though present Galactocentric distances may not be that different, a range much greater than the range spanned by the three reference open clusters. Indeed, Galactic orbits calculated by Silva \& Napiwotzki (2011) give birthplaces from the Galactic center out to nearly $14 \mathrm{kpc}$ for the sample drawn from their paper. However, plots of the $\mathrm{Mg}$ and $\mathrm{Si}$ abundances versus the estimated Galactocentric distance of a star's birthplace are too ill defined to confirm the abundance gradients obtained from in situ abundance measurements of young stars and $\mathrm{H}$ II regions. All but three of our stars have estimated birthplaces between 4 and $10 \mathrm{kpc}$. The failure to reproduce the observed abundance gradients may be attributable to uncertainties in the locations of the birthplaces and to the contamination of the stars in the BSS by $\mathrm{Mg}$ and $\mathrm{Si}$ from the SN. 


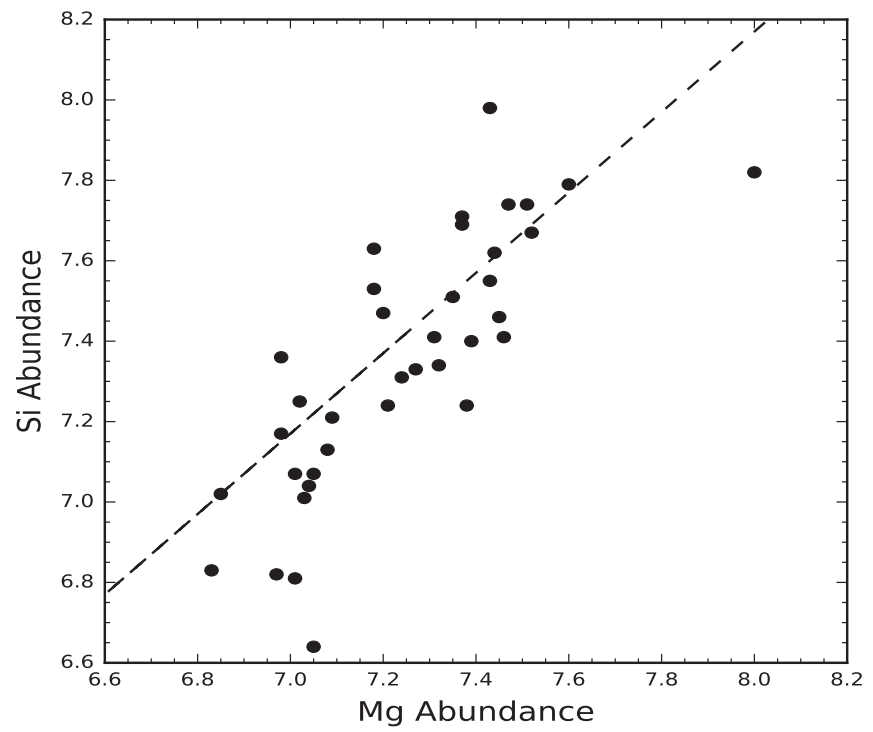

Figure 4. $\mathrm{Mg}$ and $\mathrm{Si}$ abundances from Table 3 . The straight line corresponds to an $\mathrm{Mg} / \mathrm{Si}$ ratio of -0.17 dex normalized to the standard abundances adopted by Hunter et al. (2009).

Scatter in Figure 4 about the linear relation is consistent with $\mathrm{Mg}$ and $\mathrm{Si}$ abundance uncertainties and with the spread in $\mathrm{Mg}$ abundances within each of the open clusters (see Figure 7, for example). Presence of the linear correlation and a constant $\mathrm{Mg} / \mathrm{Si}$ ratio are qualitatively expected from the similar nucleosynthetic yields of $\mathrm{Mg}$ and $\mathrm{Si}$ from $\mathrm{SNe}$ II, which are surely the controlling influences on the Galactic abundance gradient whatever the influence of the myriad other factors (e.g., the initial mass function, the star formation rate, Galactic infall etc.) entering recipes for Galactic chemical evolution.

\section{2. $C$ and $N$}

Hunter et al. (2009) found that among the B stars in the three reference clusters, the $\mathrm{C}$ and $\mathrm{N}$ abundances were independent of position on a star's evolutionary track except that half of the few supergiants were enriched in $\mathrm{CN}$-cycled material. Similarly, Lyubimkov et al. (2013) found normal C and N abundances in a sample of 22 slightly evolved B stars (i.e., no supergiants) except for two possibly mixed stars. Thus the expectation is that the majority of the B stars would have preserved their initial $\mathrm{C}$ and $\mathrm{N}$ abundances before undergoing conversion to a runaway star. The likely corollary of this expectation is that alterations to the $\mathrm{C}$ and $\mathrm{N}$ abundances may be clues to the process creating the runaway star but that internal $\mathrm{CN}$ cycling and mixing to the surface may have occurred independently of formation as a runaway star.

As noted above, the $\mathrm{C}$ abundances in Table 3 are provided only for the stars observed with FEROS. Figure 5 shows the $\mathrm{C}$ and $\mathrm{N}$ abundances for this minority. In Table 3 the $\mathrm{C}$ and $\mathrm{N}$ abundances for EC 05582-5816, unlike for other stars, are taken from an analysis with the $\mathrm{Si}$ abundance fixed at 7.4 but, as noted above, the $\mathrm{C}$ and $\mathrm{N}$ abundances are expected to be independent of this assumption concerning the Si abundance; the high $v \sin i$ of EC 05582-5816 precluded the use of the Si III multiplet to determine the microturbulence. The $\mathrm{C}$ and $\mathrm{N}$ abundances are tightly correlated with all stars showing a similar $\mathrm{C} / \mathrm{N}$ to within the measurement uncertainties. In addition, the three Galactic clusters analyzed by Hunter et al. (2009) have mean $\mathrm{C}$ and $\mathrm{N}$ abundances falling within the band

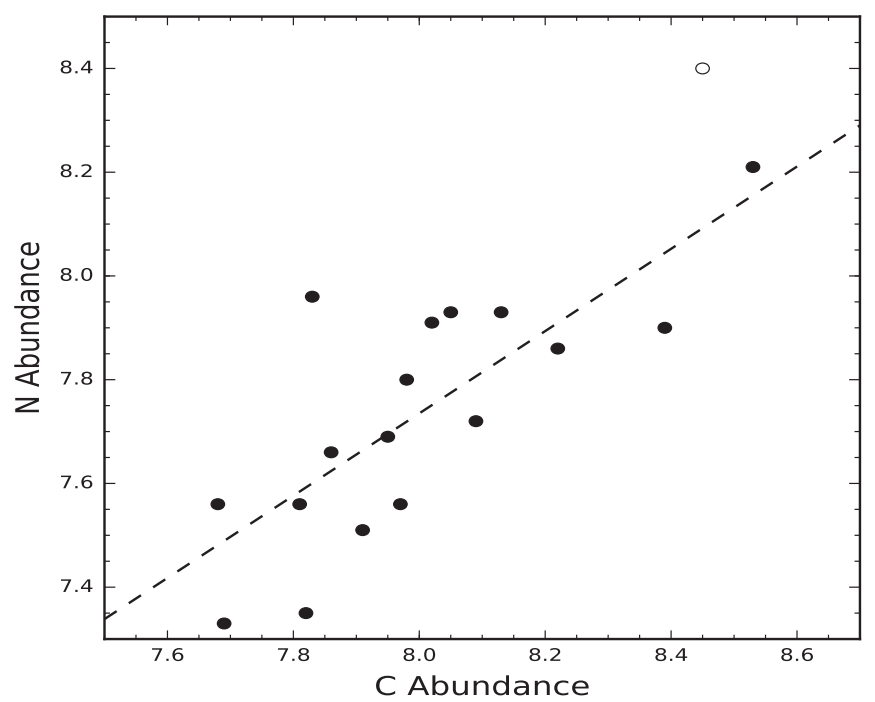

Figure 5. $\mathrm{C}$ and $\mathrm{N}$ abundances plotted for the FEROS sample of runaways. Abundances are taken from Table 3 . The straight line, a least-squares fit, shows that the $\mathrm{C}$ and $\mathrm{N}$ abundances are related over a range of about $1 \mathrm{dex}$ with a scatter consistent with measurement uncertainties-i.e., there are no stars heavily contaminated with $\mathrm{CN}$-cycled products; that is, stars that are C-poor and N-rich. Data for EC 05582-5816 (open circle) are taken from the analysis in which the $\mathrm{Si}$ abundance is fixed at 7.4.

defined by the runaway stars; that is, mean abundances of $8.00 \pm 0.19$ and $7.62 \pm 0.12$ for $\mathrm{C}$ and $\mathrm{N}$, respectively, for all nonsupergiants in the three Galactic clusters with these abundances provided by analyses assuming that $\mathrm{Si}$ has its standard abundance.

These carbon abundances for the subset of our runaway stars show no evidence for a runaway star enriched in $\mathrm{CN}$-cycled material, i.e., a low $\mathrm{C}$ abundance paired with a high $\mathrm{N}$ abundance such that the total number of $\mathrm{C}$ and $\mathrm{N}$ atoms is conserved. Very severe contamination with $\mathrm{CN}$-cycled material would result in an $\mathrm{N}$ enrichment of up to $0.5-0.6 \mathrm{dex}$ and a severe depletion of $\mathrm{C}$. No such stars are seen in Figure 5. The presence of $\mathrm{CN}$-cycled material may occur in the B star prior to its conversion to a runaway star and is thus not a determining signature of a process resulting in a runaway star.

\section{3. $\mathrm{N}$ and $\mathrm{Mg}$}

To extend the search for abundance anomalies to the complete sample, we examine next the relationship between the $\mathrm{N}$ and the $\mathrm{Mg}$ abundances. Figure 6, drawing on Table 3, compares the $\mathrm{N}$ and the $\mathrm{Mg}$ abundances with a distinction made according to surface gravity (i.e., evolutionary status) with stars with $\log g<3.2$ (i.e., supergiants) represented by open circles and stars of higher surface gravity (i.e., mainsequence and slightly evolved stars) represented by filled circles. Figure 7 shows the $\mathrm{N}$ and $\mathrm{Mg}$ abundances for B stars belonging to the cluster NGC 3293, the best represented of the three clusters studied by Hunter et al. (2009).

With the possible exception of $\mathrm{N}$ enrichment from internal mixing or mass loss, one expects the cluster's stars in Figure 7 to share the same $\mathrm{N}$ and certainly the same $\mathrm{Mg}$ abundance. Thus, the scatter in the $\mathrm{N}$ and the $\mathrm{Mg}$ abundances in Figure 7 represents the measurement uncertainties that, thanks to the similarity of analytical techniques, will be a very close approximation to the uncertainties affecting the points in Figure 6. (One star-a supergiant-in NGC 3293 appears $\mathrm{N}$-enriched, but this star is not $\mathrm{C}$-depleted.) A comparison of 


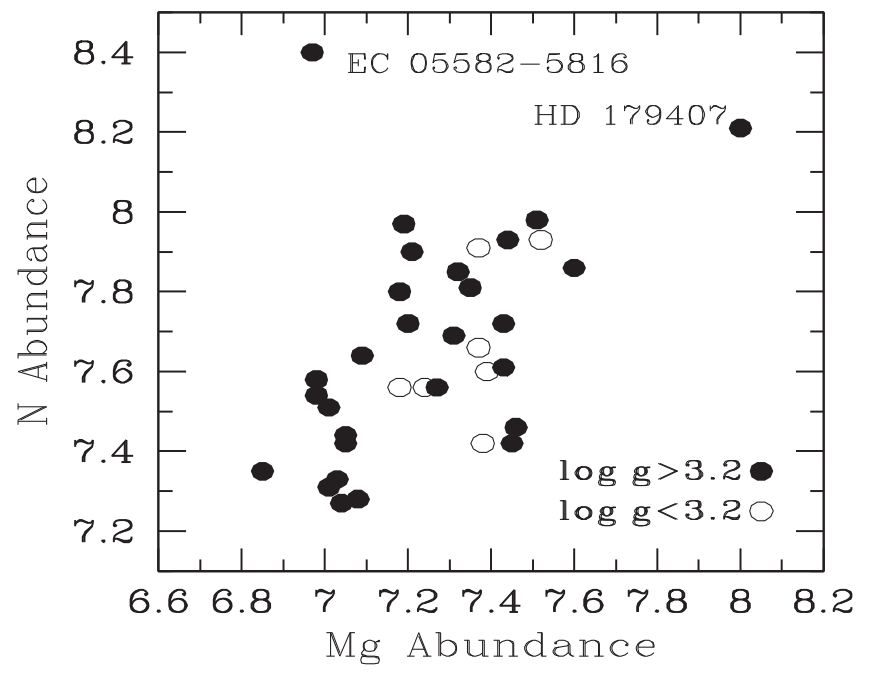

Figure 6. $\mathrm{Mg}$ and $\mathrm{N}$ abundances from Table 3 with stars distinguished by their surface gravity: $\log g>3.2$, shown as filled circles, and $\log g<3.2$, shown as unfilled circles. Two outliers are identified-EC 05582-5816 and HD 179407 - and are discussed in the text.

Figures 6 and 7 shows the spread shown by NGC 3293's B stars and hence we conclude that the runaways show an intrinsic star-to-star difference in composition (i.e., C, $\mathrm{N}, \mathrm{Mg}$, and $\mathrm{Si}$ abundances but with similar abundance ratios), which we attribute to differences in a star's birthplace, with the Galactic abundance gradient being a very likely controlling factor.

EC 05582-5816 and HD 179407 appear as outliers in Figure 6 . The two outliers have similar compositions except for their $\mathrm{Mg}$ abundances and both appear related to the inner Galaxy. Silva \& Napiwotzki (2011) estimate EC 05582-5816's birthplace at $2 \mathrm{pc}$ from the Galactic center. HD 179407 is presently at $R_{G}=3.5 \mathrm{kpc}$ according to Smartt et al. (1997). The pair have very similar $\mathrm{C}$ and $\mathrm{N}$ abundances (Table 3 ) but differ substantially in $\mathrm{Mg}$ with an abundance of 8.0 for HD 179407 but 7.0 for EC 05582-5816. The Si abundance of HD 179407 is consistent with its Mg abundance. For EC 055825816 , the present analysis assumes an $\mathrm{Si}$ abundance of 7.4. Galactic abundance gradients likely account for the high abundances found for HD 179407. There is a remarkable similarity in composition between EC 05582-5816 and the B9 III secondary of the BH binary V4641 Sgr. This B star has $[\mathrm{Fe} / \mathrm{H}]=0$ and normal abundances of $\mathrm{C}, \mathrm{O} \mathrm{Mg}, \mathrm{Al}, \mathrm{Si}$, and $\mathrm{Ti}$ but high $\mathrm{N}$ and $\mathrm{Na}$ overabundances $([\mathrm{N} / \mathrm{H}]=$ $[\mathrm{Na} / \mathrm{H}]=+0.8$ ) (Sadakane et al. 2006). If placed in Figure 6, it would provide a third outlier and fall near the two existing outliers. Reobservation and reanalysis of EC 05582-5816, a rapid rotator, is to be encouraged and extended to additional elements.

\subsection{Formation Mechanisms and Abundance Anomalies?}

$\mathrm{N}, \mathrm{Mg}$, and $\mathrm{Si}$ abundances across the sampled runaway $\mathrm{B}$ stars are similar to those seen in B stars in Galactic clusters. The spread in the $\mathrm{C}, \mathrm{N}, \mathrm{Mg}$, and $\mathrm{Si}$ abundances and the nearly uniform abundance ratios $\mathrm{C} / \mathrm{N} / \mathrm{Mg} / \mathrm{Si}$ among runaway stars in our sample results from the combination of two facts: (1) the stars' birthplaces sample a wide range in Galactocentric distances, and (2) the abundances in star-forming regions decline with increasing Galactocentric distance at a rate of approximately $0.05 \mathrm{dex} \mathrm{kpc}^{-1}$. Within our sample there are no

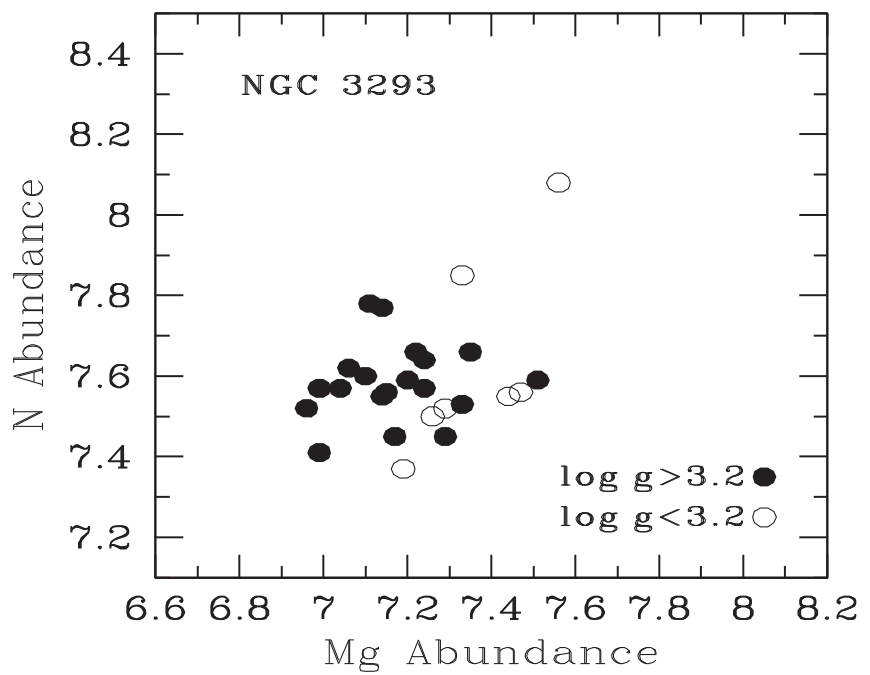

Figure 7. $\mathrm{Mg}$ and $\mathrm{N}$ abundances for B stars in the open cluster NGC 3293 with data from Hunter et al. (2009). Stars are distinguished by their surface gravity - see caption to Figure 6.

certain outliers with abundance peculiarities. Thus the conclusion is that the two scenarios-BSS and CES-capable of ejecting B (and other) stars from the Galactic disk into the Galactic halo do not as a rule change the surface chemical composition of the runaway star, as sampled by $\mathrm{C}, \mathrm{N}, \mathrm{Mg}$, and $\mathrm{Si}$. This conclusion from our non-LTE analysis confirms earlier LTE analyses in which the compositions of a runaway star and a comparable B star in the Galactic disk are compared-see, e.g., Martin (2004). Although changes may occur in rare cases, the general conclusion is both disappointing and challenging: disappointing in that the composition, a readily obtainable quantity, appears not to be a discriminant between competing ejection mechanisms; challenging in that other observable quantities now have to been relied on to identify the principal ejection mechanism across a sample of runaway stars and for individual runaway stars. Possible observables include projected rotational velocities and binarity.

\section{Ejection Mechanisms: Rotational and Radial Velocities}

\subsection{Projected Rotational Velocities}

The distribution of projected rotational velocities $v \sin i$ for our sample of runaways is very similar to that provided by the B stars in the three Galactic clusters observed by Hunter et al. (2009): velocities up to $300 \mathrm{~km} \mathrm{~s}^{-1}$ with a peak near $50 \mathrm{~km} \mathrm{~s}^{-1}$ and few slow rotators $\left(v \sin i \leqslant 30 \mathrm{~km} \mathrm{~s}^{-1}\right)$. Martin (2006) considered rotational velocities for his sample of runaways and found their frequency distribution to be similar to that assembled by Guthrie (1984) from "young" OB associations whose result is similar to that from the three Galactic clusters. In contrast, B stars in "old" associations and field B stars in the Galactic disk have distributions that rise with a decreasing $v \sin i$ (Wolff et al. 1982; Guthrie 1984). A more recent catalog of projected rotational velocities for 102 northern B stars is provided by Abt et al. (2002).

Tying the $v \sin i$ distribution of the runaways to the ejection mechanisms is unfortunately hampered by the absence of quantitative predictions for the BSS and the CES. In the BSS, if the runaway originates in a close binary system the runaway may be tidally locked, resulting in the rotation velocity being closely related to its orbital velocity. In turn, the ejection 
velocity is expected to be related to the orbital velocity. These ideas lead to the expectation that the ejection velocity should be positively correlated with the rotational velocity, but as Martin (2006) demonstrated, runaway stars do not show the expected correlation and therefore the BSS is not the leading producer of runaway stars. For the CES, the likelihood of ejection is plausibly higher in denser environments. On this simple premise and the match between the $v \sin i$ frequency distribution for runaways and young associations, Martin (2006) considered that it seems likely that the [CES] is the dominant mechanism behind his runaway sample and by extension ours too, which has overlap with his sample.

\subsection{Radial Velocities}

In the BSS, the resulting runaway B star will either be a single B star or form a binary system with a low-mass compact companion (e.g., an NS). A runaway that is single will be paired with a distant ejected NS or a $\mathrm{BH}$ moving in a generally opposite direction. Portegies Zwart (2000) suggests that 20\%$40 \%$ of the runaway B stars should be accompanied by an NS and thus the runaway B star should appear as a single-lined spectroscopic binary with a period of several hundred days.

In the CES, simulations (Leonard \& Duncan 1990) suggest that $10 \%$ of the runaways will be binaries composed of normal main-sequence stars and likely observable as double-lined spectroscopic binaries.

A definitive test of these predictions for the BSS and the CES will require a radial velocity long-term survey of a large sample of runaway B stars to be put up against more precise predictions about the frequency and nature of the binary populations from the two scenarios. Then, it may be possible to assess in a statistical fashion the relative production rates from the BSS and the CES. For the binaries, it should be possible to assign them to either the BSS (i.e., an NS/pulsar companion) or the CES (i.e., a normal main-sequence companion). For the runaway single stars, the attribution to the formation scenario will be difficult unless one can uncover a subtle abundance anomaly or apply a precise determination of space motions $(\text { GAIA })^{8}$ to assist in the identification of the pulsar or the original stellar association.

A definitive observational test is not possible at present. The sole radial velocity survey of high Galactic OB stars was conducted by Gies \& Bolton (1986), who concluded that runaway $\mathrm{OB}$ stars are deficient in close binaries by a factor of 2-4. Their sample of 15 confirmed runaways provided two binaries (both double-lined systems), and if five probable runways are added, the binary probability becomes $2 / 20$ or $10 \%$. The deficiency of binaries is suggested by reference to the binary fraction of $31 \%$ among normal $\mathrm{O}$ stars (Garmany et al. 1980) and of $38 \%$ among normal B stars (Abt \& Levy 1978). Four of our stars (HD 97991, 149363, 214930, and 219188) were in the Gies \& Bolton (1986) survey and declared by them to have a constant radial velocity. Martin (2003) found the runaway HD 138503 to be a double-lined spectroscopic binary, a star in Silva \& Napiwotzki's (2011) list of runaway stars. HD 1999 and HD 204076, which we observed, are double-lined binaries. Two radio surveys for pulsars reported no detections coincident with runaway OB stars (Philp et al. 1996; Sayer et al. 1996). Unfortunately, the correction for the beaming of pulsar radiation results in an

\footnotetext{
https://www.cosmos.esa.int/web/gaia/
}

uninteresting limit on the fraction of runaway stars with low-mass compact companions; Sayer et al. (1996) estimate that less than $25 \%-50 \%$ of OB runaways have an NS companion, an (1996) estimate consistent with the prediction by Portegies Zwart (2000).

Our sample can make only a modest contribution to the frequency of binary runaway stars as a test of the BSS and the CES. For the majority of our sample the star was observed once and an assessment of the radial velocity variation must come from velocities reported in the literature. Eight stars were observed at two or more telescopes and thus at different epochs, and the two or three radial velocity measurements may be intercompared and also checked against the literature. (This restricted search for velocity variations is akin to that reported by Martin (2006), who observed many runaway stars at least twice over a few days and also checked velocities against the literature.) We have defined a star with a radial velocity variation between measurements of greater than $20 \mathrm{~km} \mathrm{~s}^{-1}$ as a possible member of a binary system. This cutoff is set by an inspection of other studies of OB stars (Sana et al. 2013; Dunstall et al. 2015). In some stars, significant velocity variations arise from pulsations, which complicates the search for orbital radial velocity variations, and if neglected as a source of radial velocity variations, the fraction of spectroscopic binaries is overestimated.

Radial velocities are provided in Table 4. Of the eight stars observed twice or thrice by us, seven appear to have a constant velocity. The star with a variable radial velocity is HD 204076, which is certainly a spectroscopic binary; the UVES but not the FEROS spectrum showed double lines. HD 219188 is possibly a variable. There is a $25 \mathrm{~km} \mathrm{~s}^{-1}$ difference between our two measurements, and online catalogs give a velocity that is either close to the mean of our two or a less positive velocity: $84 \mathrm{~km} \mathrm{~s}^{-1}$ according to Silva \& Napiwotzki (2011) or $64 \mathrm{~km} \mathrm{~s}^{-1}$ according to Gontcharov (2006) and Kharchenko et al. (2007).

Inspection of Table 4 suggests that four stars observed once by us may be binaries because of a difference with radial velocities reported in the literature. EC 20140-6935 (HD 192273) was noted as a possible binary by Magee et al. (1998), who found a $45 \mathrm{~km} \mathrm{~s}^{-1}$ velocity difference between their measurement and that reported by Rolleston et al. (1997). Silva \& Napiwotzki (2011) gave the velocity as $+17 \mathrm{~km} \mathrm{~s}^{-1}$ from Rolleston et al. (1997). Our velocity is in good agreement with that by Magee et al. (1998) HD 188618 was suspected by Martin (2006) to be a binary from the velocity difference between his measurement of $29 \pm 6 \mathrm{~km} \mathrm{~s}^{-1}$ and a previous measurement of $-15 \mathrm{~km} \mathrm{~s}^{-1}$ by Duflot et al. (1998). Our $-46 \mathrm{~km} \mathrm{~s}^{-1}$ extends the velocity range. HD 179407's present and previous velocity measurements barely satisfy our $20 \mathrm{~km} \mathrm{~s}^{-1}$ condition. HIP 70205 (LP 857-24) exhibits a $300 \mathrm{~km} \mathrm{~s}^{-1}$ difference but the only previous measurement is from the RAVE survey (Kordopatis et al. 2013), which is possibly ill suited to velocity measurements of B stars that provide few lines in the RAVE bandpass. Obviously, this star deserves further attention. Finally there is HD 114569 , which has no previous velocity measurement.

It seems fair to conclude that our sample contains few spectroscopic binaries and certainly fewer than the approximately $30 \%$ provided by a sample of field B stars in the Galactic disk (Abt \& Levy 1978). Our sample is very slightly biased by the exclusion of known double-lined or even singlelined spectroscopic binaries. HD 1999 and the double-lined 
Table 4

Each Star Listed by HIP Number Along with Alternative Identifier, Radial Velocity from Our Analysis and from the Literature (with References), and Binary Status of the Star

\begin{tabular}{|c|c|c|c|c|c|}
\hline \multirow{2}{*}{ HIP } & \multirow{2}{*}{ Other } & \multicolumn{2}{|l|}{ Radial Velocity } & \multirow{2}{*}{ Reference $^{\mathrm{b}}$} & \multirow{2}{*}{ Status } \\
\hline & & $\mathrm{Us}^{\mathrm{a}}$ & Literature & & \\
\hline 2702 & HD 3175 & $-13 \pm 2(\mathrm{~F})$ & $-16 \pm 3$ & $\mathrm{R} 3$ & Single \\
\hline 3812 & $\mathrm{CD}-56152$ & $14 \pm 8(\mathrm{U})$ & $19 \pm 10$ & $\mathrm{R} 2$ & Single \\
\hline 7873 & HD 10747 & $-9 \pm 2(\mathrm{~F})$ & $-12 \pm 2$ & $\mathrm{R} 1$ & Single \\
\hline 13489 & HD 18100 & $80 \pm 7(\mathrm{~F})$ & $76 \pm 3$ & $\mathrm{R} 1$ & Single \\
\hline 16758 & HD 22586 & $99 \pm 1(\mathrm{~F})$ & $97 \pm 2$ & R3 & Single \\
\hline 45563 & HD 78584 & $-120 \pm 6(\mathrm{~T})$ & $-125 \pm 2$ & $\mathrm{R} 1$ & Single \\
\hline 55051 & HD 97991 & $31 \pm 3(\mathrm{U})$ & $26 \pm 3$ & R3 & Single \\
\hline 56322 & HD 100340 & $253 \pm 10(\mathrm{~T}), 263 \pm 4(\mathrm{U})$ & $254 \pm 9$ & $\mathrm{R} 2$ & Single \\
\hline 60615 & $\mathrm{BD}+362268$ & $31 \pm 4(\mathrm{~T})$ & $31 \pm 7$ & $\mathrm{R} 3$ & Single \\
\hline 61431 & HD 109399 & $-43 \pm 3(\mathrm{~F})$ & $-49 \pm 2$ & $\mathrm{R} 1$ & Single \\
\hline 64458 & HD 114569 & $104 \pm 2(\mathrm{~F})$ & $\cdots$ & & Unknown \\
\hline 67060 & HD 119608 & $31 \pm 1(\mathrm{~F})$ & $26 \pm 4$ & $\mathrm{R} 1$ & Single \\
\hline 68297 & HD 121968 & $17 \pm 9(\mathrm{~T}), 29 \pm 3(\mathrm{U})$ & $28 \pm 2$ & $\mathrm{R} 4$ & Single \\
\hline 70205 & LP 857-24 & $243 \pm 4(\mathrm{~F})$ & $-54 \pm 2$ & R5 & Binary? \\
\hline 70275 & HD 125924 & $244 \pm 1(\mathrm{~T})$ & $239 \pm 2$ & $\mathrm{R} 4$ & Single \\
\hline 79649 & HD 146813 & $21 \pm 2(\mathrm{~T})$ & $19 \pm 6$ & $\mathrm{R} 4$ & Single \\
\hline 81153 & HD 149363 & $145 \pm 3(\mathrm{~T}), 146 \pm 3(\mathrm{U}), 144 \pm 3(\mathrm{~F})$ & $141 \pm 2$ & $\mathrm{R} 1$ & Single \\
\hline 85729 & HD 158243 & $-63 \pm 2(\mathrm{~F})$ & $-64 \pm 3$ & $\mathrm{R} 1$ & Single \\
\hline 91049 & HD 171871 & $-64 \pm 1(\mathrm{~T})$ & $-62 \pm 5$ & $\mathrm{R} 1$ & Single \\
\hline 92152 & HD 173502 & $49 \pm 1(\mathrm{~F})$ & $68 \pm 4$ & $\mathrm{R} 1$ & Single? \\
\hline 94407 & HD 179407 & $-120 \pm 4(\mathrm{~F})$ & $-119 \pm 5$ & R6 & Single \\
\hline 96130 & HD 183899 & $-46 \pm 2(\mathrm{~F})$ & $-45 \pm 5$ & $\mathrm{R} 1$ & Single \\
\hline 98136 & HD 188618 & $46 \pm 4(\mathrm{~F})$ & $-15 \pm 5$ & $\mathrm{R} 1$ & Binary $?^{\mathrm{c}}$ \\
\hline 101328 & HD 195455 & $19 \pm 7(\mathrm{~F}), 10 \pm 6(\mathrm{U})$ & $10 \pm 6$ & $\mathrm{R} 3$ & Single \\
\hline 105912 & HD 204076 & $0 \pm 3(\mathrm{~F}), 14 \pm 2(\mathrm{U})$ & $-17 \pm 7$ & $\mathrm{R} 2$ & Binary \\
\hline 107027 & HD 206144 & $122 \pm 5(\mathrm{~F}), 121 \pm 2(\mathrm{U})$ & $117 \pm 8$ & $\mathrm{R} 4$ & Single \\
\hline 109051 & HD 209684 & $82 \pm 2(\mathrm{U})$ & $72 \pm 8$ & R4 & Single \\
\hline 111563 & HD 214080 & $16 \pm 2(\mathrm{~F})$ & $12 \pm 4$ & R6 & Single \\
\hline 112022 & HD 214930 & $-60 \pm 4(\mathrm{~T})$ & $-63 \pm 2$ & $\mathrm{R} 3$ & Single \\
\hline 112482 & HD 215733 & $-6 \pm 6(T)$ & $-15 \pm 2$ & $\mathrm{R} 3$ & Single \\
\hline 113735 & HD 217505 & $-17 \pm 1(\mathrm{~F})$ & $-31 \pm 10$ & $\mathrm{R} 2$ & Single \\
\hline 114690 & HD 219188 & $73 \pm 19(\mathrm{~F}), 98 \pm 19(\mathrm{~T})$ & $64 \pm 3$ & $\mathrm{R} 1$ & Single ${ }^{\mathrm{d}}$ \\
\hline 115347 & HD 220172 & $26 \pm 2(\mathrm{~F})$ & $29 \pm 3$ & R6 & Single \\
\hline 115729 & HD 220787 & $26 \pm 2(\mathrm{~F})$ & $26 \pm 3$ & $\mathrm{R} 4$ & Single $^{e}$ \\
\hline$\cdots$ & EC 05582-5816 & $85 \pm 13(\mathrm{~F})$ & $81 \pm 10$ & $\mathrm{R} 2$ & Single \\
\hline$\cdots$ & EC 13139-1851 & $15 \pm 4(\mathrm{~F})$ & $23 \pm 10$ & $\mathrm{R} 2$ & Single \\
\hline$\cdots$ & EC 20140-6935 & $-24 \pm 2(\mathrm{U})$ & $17 \pm 10$ & $\mathrm{R} 2$ & Binary $^{f}$ \\
\hline$\cdots$ & PB 5418 & $147 \pm 3(\mathrm{U})$ & $152 \pm 10$ & $\mathrm{R} 2$ & Single \\
\hline$\cdots$ & PHL 159 & $87 \pm 2(\mathrm{U})$ & $88 \pm 3$ & $\mathrm{R} 2$ & Single \\
\hline
\end{tabular}

Notes.

${ }^{\mathrm{a}}$ Spectrograph used for the observation: F-Feros, T-Tull, and U-UVES.

b References: R1—Gontcharov (2006), R2—Silva \& Napiwotzki (2011), R3-Kharchenko et al. (2004), R4-Martin (2006), R5-Kordopatis et al. (2013), R6-Kilkenny \& Hill (1975).

${ }^{c}$ Martin (2006) obtained the velocity $29 \pm 6 \mathrm{~km} \mathrm{~s}^{-1}$ and also quoted $-15 \mathrm{~km} \mathrm{~s}^{-1}$ from Duflot et al. (1998).

${ }^{\mathrm{d}} \mathrm{R} 2$ gives the velocity as $84 \mathrm{~km} \mathrm{~s}^{-1}$.

${ }^{\mathrm{e}} \mathrm{R} 4$ also gives velocities of $24.9 \pm 1.5 \mathrm{~km} \mathrm{~s}^{-1}$ from Barbier-Brossat \& Figon (2000) and $26.5 \pm 2.4 \mathrm{~km} \mathrm{~s}^{-1}$ from Behr (2003).

${ }^{\mathrm{f}}$ See Section 6.2.

eclipsing star HD 138503 (Martin 2003) are the only binary stars observed, but they are not in Table 4 . With the present understanding of the formation of single and double runaway stars by the BSS and the CES, it is not possible to interpret the low fraction of spectroscopic binaries as a pointer to the more important formation mechanism.

\section{Concluding Remarks}

Our sample of runaway stars is dominated by B stars that have been ejected from sites of recent star formation in the
Galactic disk. Our non-LTE analysis of their $\mathrm{C}, \mathrm{N}, \mathrm{Mg}$, and $\mathrm{Si}$ abundances and published analyses of these elemental abundances by the same non-LTE procedures in B stars in three open clusters in the Galactic disk show no abundance anomalies among the runaways that may be attributed to either of the leading two mechanisms (the BSS and the CES) obviously capable of producing runaways. The spread in abundances over a range of about 1 dex surely reflects the range in the Galactocentric distance of the birthplaces of the stars before they are ejected into the halo and the presence of an abundance gradient in the Galaxy. Consideration of either the 
projected rotational velocities or the radial velocities cannot provide a determination of the relative probabilities of the BSS and the CES in providing the runaway B stars. But an intensive search for binaries among the B runaway population may yet shed light on the relative contributions of the BSS and the CES. The ability to identify runaway stars formed by the BSS will be enhanced by extending the non-LTE analysis to other elements, notably $\mathrm{O}$ and $\mathrm{S}$, which appear to have large excesses in $[\mathrm{X} / \mathrm{Fe}]$ in the (few) LMXB secondaries analyzed for this pair of elements.

We thank R. Napiwotzki, the referee, for constructive reports. D.L.L. thanks A.B.S. Reddy for his help in preparing the manuscript for submission. C.M.M. is grateful to the Department of Education and Learning in Northern Ireland and Queen's University Belfast for the award of a research studentship. This work was supported by the Science and Technology Faculty Council and the UIC. D.L.L. thanks the Robert A. Welch Foundation of Houston, Texas, for support through grant F-634. This work was partly funded by the Director General Discretionary fund at ESO. ESO observations collected via programme IDs 091.D-0061(A) for FEROS and 093.D-0302(A) for UVES.

\section{References}

Abt, H. A., Levato, H., \& Grosso, M. 2002, ApJ, 573, 359

Abt, H. A., \& Levy, S. G. 1978, ApJS, 36, 241

Aerts, C., De Cat, P., De Ridder, J., et al. 2006, A\&A, 449, 305

Ballester, P., Modigliani, A., Boitquin, O., et al. 2000, Msngr, 101, 31

Barbier-Brossat, M., \& Figon, P. 2000, A\&AS, 142, 217

Behr, B. B. 2003, ApJS, 149, 101

Blaauw, A. 1961, BAN, 15, 265

Blaauw, A. 1993, in ASP Conf. Ser. 35, Massive Stars: Their Lives in the Interstellar Medium, ed. J. P. Cassinelli \& E. B. Churchwell (San Francisco, CA: ASP), 207

Blaauw, A., \& Morgan, W. W. 1954, ApJ, 119, 625

Brott, I., de Mink, S. E., Cantiello, M., et al. 2011, A\&A, 530, A115

Brown, W. R. 2015, ARA\&A, 53, 15

Daflon, S., \& Cunha, K. 2004, ApJ, 617, 1115

Daflon, S., Cunha, K., de la Reza, R., Holtzman, J., \& Chiappini, C. 2009, AJ, 138, 1577

de Bruijne, J. H. J., \& Eilers, A.-C. 2012, A\&A, 546, A61

de Zeeuw, T., Hoogerwerf, R., \& de Bruijne, J. 2001, in ASP Conf. Ser. 228,

Dynamics of Star Clusters and the Milky Way, ed. S. Deiters et al. (San Francisco, CA: ASP), 201

Dekker, H., D’Odorico, S., Kaufer, A., Delabre, B., \& Kotzlowski, H. 2000, Proc. SPIE, 4008, 534

Duflot, M., Figon, P., \& Meyssonnier, N. 1998, yCat, 3190

Dufton, P. L., Ryans, R. S. I., Trundle, C., et al. 2005, A\&A, 434, 1125

Dunstall, P. R., Dufton, P. L., Sana, H., et al. 2015, A\&A, 580, A93

Dyson, J. E., \& Hartquist, T. W. 1983, MNRAS, 203, 1233

Fraser, M., Dufton, P. L., Hunter, I., \& Ryans, R. S. I. 2010, MNRAS, 404, 1306

Garmany, C. D., Conti, P. S., \& Massey, P. 1980, ApJ, 242, 1063

Gies, D. R., \& Bolton, C. T. 1986, ApJS, 61, 419

Gontcharov, G. A. 2006, AstL, 32, 759

González Hernández, J. I., Rebolo, R., \& Israelian, G. 2008, A\&A, 478, 203

Greenstein, J. L., \& Sargent, A. I. 1974, ApJS, 28, 157

Guthrie, B. N. G. 1984, MNRAS, 210, 159

Gvaramadze, V. V., Gualandris, A., \& Portegies Zwart, S. 2009, MNRAS, 396,570

Heber, U., Edelmann, H., Napiwotzki, R., Altmann, M., \& Scholz, R.-D. 2008, A\&A, 483, L21
Hoffer, J. B. 1983, AJ, 88, 1420

Hoogerwerf, R., de Bruijne, J. H. J., \& de Zeeuw, P. T. 2001, A\&A, 365, 49 Huang, Y., Liu, X.-W., Zhang, H.-W., et al. 2015, RAA, 15, 1240

Hubeny, I. 1988, CoPhC, 52, 103

Hubeny, I., Heap, S. R., \& Lanz, T. 1998, in ASP Conf. Ser. 131, Properties of Hot Luminous Stars, ed. I. Howarth (San Francisco, CA: ASP), 108

Hubeny, I., \& Lanz, T. 1995, ApJ, 439, 875

Hunter, I., Brott, I., Langer, N., et al. 2009, A\&A, 496, 841

Hunter, I., Brott, I., Lennon, D. J., et al. 2008, ApJL, 676, L29

Hunter, I., Dufton, P. L., Smartt, S. J., et al. 2007, A\&A, 466, 277

Irrgang, A., Przybilla, N., Heber, U., Nieva, M. F., \& Schuh, S. 2010, ApJ, 711,138

Kaufer, A., Stahl, O., Tubbesing, S., et al. 1999, Msngr, 95, 8

Keenan, F. P., Dufton, P. L., \& McKeith, C. D. 1982, MNRAS, 200, 673

Kharchenko, N. V., Piskunov, A. E., \& Scholz, R.-D. 2004, AN, 325, 439

Kharchenko, N. V., Scholz, R.-D., Piskunov, A. E., Röser, S., \& Schilbach, E. 2007, AN, 328, 889

Kilkenny, D., \& Hill, P. W. 1975, MNRAS, 172, 649

Kordopatis, G., Gilmore, G., Steinmetz, M., et al. 2013, AJ, 146, 134

Lanz, T., \& Hubeny, I. 2007, ApJS, 169, 83

Leonard, P. J. T. 1989, AJ, 98, 217

Leonard, P. J. T. 1993, in ASP Conf. Ser. 45, Luminous High-Latitude Stars, ed. D. D. Sasselov (San Francisco, CA: ASP), 360

Leonard, P. J. T., \& Duncan, M. J. 1990, AJ, 99, 608

Leonard, P. J. T., \& Fahlman, G. G. 1991, AJ, 102, 994

Luck, R. E., \& Lambert, D. L. 2011, AJ, 142, 136

Lyubimkov, L. S., Lambert, D. L., Poklad, D. B., Rachkovskaya, T. M., \& Rostopchin, S. I. 2013, MNRAS, 428, 3497

Lyubimkov, L. S., Rostopchin, S. I., Rachkovskaya, T. M., Poklad, D. B., \& Lambert, D. L. 2005, MNRAS, 358, 193

Magee, H. R. M., Dufton, P. L., Keenan, F. P., et al. 1998, A\&A, 338, 85

Martin, J. C. 2003, PASP, 115, 49

Martin, J. C. 2004, AJ, 128, 2474

Martin, J. C. 2006, AJ, 131, 3047

McDonald, I., Zijlstra, A. A., \& Boyer, M. L. 2012, MNRAS, 427, 343

McEvoy, C. M., Dufton, P. L., Evans, C. J., et al. 2015, A\&A, 575, A70

Mdzinarishvili, T. G., \& Chargeishvili, K. B. 2005, A\&A, 431, L1

Nieva, M. F., \& Przybilla, N. 2006, ApJL, 639, L39

Nieva, M. F., \& Przybilla, N. 2008, A\&A, 481, 199

Nieva, M.-F., \& Simón-Díaz, S. 2011, A\&A, 532, A2

Philp, C. J., Evans, C. R., Leonard, P. J. T., \& Frail, D. A. 1996, AJ, 111, 1220

Portegies Zwart, S. F. 2000, ApJ, 544, 437

Poveda, A., Ruiz, J., \& Allen, C. 1967, BOTT, 4, 86

Przybilla, N., Fernanda Nieva, M., Heber, U., \& Butler, K. 2008, ApJL, 684, L103

Puls, J., Urbaneja, M. A., Venero, R., et al. 2005, A\&A, 435, 669

Rolleston, W. R. J., Hambly, N. C., Dufton, P. L., et al. 1997, MNRAS, 290, 422

Rolleston, W. R. J., Smartt, S. J., Dufton, P. L., \& Ryans, R. S. I. 2000, A\&A 363,537

Ryans, R. S. I., Dufton, P. L., Mooney, C. J., et al. 2003, A\&A, 401, 1119

Sadakane, K., Arai, A., Aoki, W., et al. 2006, PASJ, 58, 595

Sana, H., de Koter, A., de Mink, S. E., et al. 2013, A\&A, 550, A107

Santolaya-Rey, A. E., Puls, J., \& Herrero, A. 1997, A\&A, 323, 488

Sayer, R. W., Nice, D. J., \& Kaspi, V. M. 1996, ApJ, 461, 357

Shaver, P. A., McGee, R. X., Newton, L. M., Danks, A. C., \& Pottasch, S. R. 1983, MNRAS, 204, 53

Silva, M. D. V., \& Napiwotzki, R. 2011, MNRAS, 411, 2596

Simón-Díaz, S., Herrero, A., Uytterhoeven, K., et al. 2010, ApJL, 720, L174

Smartt, S. J., Dufton, P. L., \& Lennon, D. J. 1997, A\&A, 326, 763

Tetzlaff, N., Neuhäuser, R., \& Hohle, M. M. 2011, MNRAS, 410, 190

Tobin, W. 1987, in IAU Coll. 95: Second Conf. on Faint Blue Stars, ed. A. G. D. Philip, D. S. Hayes, \& J. W. Liebert (Schenectady, NY: L. Davis Press), 149

Trundle, C., Dufton, P. L., Hunter, I., et al. 2007, A\&A, 471, 625

Tull, R. G., MacQueen, P. J., Sneden, C., \& Lambert, D. L. 1995, PASP, 107, 251

Wolff, S. C., Edwards, S., \& Preston, G. W. 1982, ApJ, 252, 322

Zwicky, F. 1957, Morphological Astronomy (Berlin: Springer) 